EUROPEAN ORGANIZATION FOR NUCLEAR RESEARCH

CERN-EP/98-193

4.12.1998

\title{
ELECTRIC MONOPOLE TRANSITIONS FROM LOW ENERGY EXCITATIONS IN NUCLEI
}

\author{
J.L.Wood ${ }^{1}$, E.F.Zganjar ${ }^{2}$, C.De Coster $^{3,4}$ and K.Heyde ${ }^{3,5}$ \\ ${ }^{1}$ School of Physics, Georgia Institute of Technology, Atlanta, Georgia 30332-0430 (USA) \\ ${ }^{2}$ Department of Physics and Astronomy, Louisiana State University, Baton Rouge, Louisiana 70803 (USA) \\ ${ }^{3}$ Institute for Theoretical Physics, Vakgroep Subatomaire en Stralingsfysica, Proeftuinstraat 86, B-9000 Gent \\ (Belgium) \\ ${ }^{4}$ Postdoctoral fellow of the Fund for Scientific Research-Flanders (Belgium) \\ ${ }^{5}$ Present address : EP-ISOLDE, CERN CH 1211, Geneva 23 (Switzerland)
}

\begin{abstract}
Electric monopole (E0) properties are studied across the entire nuclear mass surface. Besides an introductory discussion of various model results (shell model, geometric vibrational and rotational models, algebraic models), we point out that many of the largest E0 transition strengths, $\rho^{2}(\mathrm{E} 0)$, are associated with shape mixing. We discuss in detail the manifestation of E0 transitions and present extensive data for : single-closed shell nuclei, vibrational nuclei, well-deformed nuclei, nuclei that exhibit sudden ground-state changes, and nuclei that exhibit shape coexistence and intruder states. We also give attention to light nuclei, odd-A nuclei, and illustrate a suggested relation between $\rho^{2}(\mathrm{E} 0)$ and isotopic shifts.
\end{abstract}

\section{(ISOLDE GENERAL)}

submitted to Nuclear Physics A 


\section{Introduction}

Electric monopole transition strengths reflect the off-diagonal matrix elements of the E0 operator. The E0 operator (see, e.g. ${ }^{1-4}$ ) can be expressed in terms of single-nucleon degrees of freedom as

$$
\hat{T}(E 0)=\sum_{k} e_{k} r_{k}^{2}
$$

where $e_{k}$ is the effective charge of the $k$ th nucleon and $\vec{r}_{k}$ is its position relative to the center of mass of the nucleus. The diagonal matrix elements of $\hat{T}(\mathrm{E} 0)$ are directly related to mean-square charge radii. Radii are well known to reveal shape changes in nuclei; these are normally expressed as isotope shifts or as isomer shifts (see, e.g., ${ }^{5}$ ). In the present study we explore the relationship between E0 transition strength, $\rho^{2}(\mathrm{E} 0)$, and shape isomerism (or shape coexistence, see, e.g., ${ }^{5}$ ) in nuclei.

The association of E0 transitions with shape coexistence is a relatively recent development ${ }^{3,6}$. Some preliminary surveys have been made ${ }^{7-9}$, but the association between $\rho^{2}(\mathrm{E} 0)$ and different shapes was only qualitative. Here we undertake a quantitative approach. Specifically we investigate the connection between $\rho^{2}(\mathrm{E} 0)$ and differences in $\left\langle r^{2}\right\rangle$ and differences in $\left\langle\beta^{2}\right\rangle$, where $\beta$ is the quadrupole deformation, between coexisting shapes.

To put the present investigation in perspective, we consider a variety of model estimates of $\rho^{2}(\mathrm{E} 0)$. We present comparisons of shell model and collective model (both "geometric" and "algebraic") estimates of $\rho^{2}(\mathrm{E} 0)$ with nuclei which are considered to be good examples of the shell model and of the different collective types. We argue that all the cases of large $\rho^{2}(\mathrm{E} 0)$ are consistent with a shape-coexistence origin. Conversely, we show that the $\rho^{2}(\mathrm{E} 0)$ values found in nuclei which are good examples of the shell model and of the different collective types are not large.

The focus of the work, as the title implies, is a critical assessment of the connection between strong E0 transitions and shape coexistence and elementary models of nuclear structure. We do not attempt an exhaustive review of all aspects of E0 transitions. We refer the reader to the latest in a series of papers by Voinova ${ }^{10,11}$ for a detailed coverage of the literature up to 1986 and E0 transitions viewed from a broader perspective.

\section{E0 Transitions : some general properties}

\section{A. Introduction}

The E0 operator, $\hat{T}(\mathrm{E} 0)$, is defined in Equation (1). This operator couples the nucleus to the electromagnetic field and gives rise to two-photon emission and internal-pair (IP) formation. It also couples the nucleus to the atomic electrons and gives rise to the internal-conversion (IC) process. Two-photon emission is possible at all energies, but is extremely improbable. Decay by IP formation only becomes possible above $1.022 \mathrm{MeV}$. For example, the $1.761 \mathrm{MeV} 0^{+}$excited state in ${ }^{90} \mathrm{Zr}$ (which is the first excited state in this nucleus) decays ${ }^{12,13}$ with probability $P_{I C}: P_{I P}: P_{2 \phi}=0.70: 0.30: 10^{-4}$. The IC process for E0 occurs primarily through the $1 \mathrm{~s}(\mathrm{~K})$ and $2 \mathrm{~s}\left(\mathrm{~L}_{1}\right)$ shells. Typical probabilities ${ }^{14-16}$ for IC in the various atomic subshells are $P_{K}: P_{L_{1}}: P_{L_{2}}: P_{L_{3}}: P_{M_{1}}=0.83: 0.13: 10^{-3}: 10^{-9}: 0.04$.

The E0 transition rate, $\frac{1}{\tau(E 0)}$, is defined ${ }^{3}$ by

$$
\frac{1}{\tau(E 0)}=\rho_{f_{i}}^{2}\left(\Omega_{K}+\Omega_{L_{1}}+\cdots+\Omega_{I P}\right),
$$

where

$$
\rho_{f i}^{2}=\left|\frac{\left\langle f\left|\sum_{k} e_{k} r_{k}^{2}\right| i\right\rangle}{e R^{2}}\right|^{2},
$$

and the $\Omega$ 's are "electronic" (non-nuclear) factors, $e$ is the unit of electrical charge, and $R$ is the nuclear radius,

$$
R \simeq 1.2 A^{1 / 3} \mathrm{fm}
$$

Tabulations of $\Omega_{K}, \Omega_{L_{1}}$ are given by Hager and Seltzer ${ }^{17}$ and Bell et al. ${ }^{14}$ and some graphs are given in ${ }^{16}$. A quick method for computing $\Omega_{K}$ and $\Omega_{L_{1}}$ is given by Kantele ${ }^{4}$. Tabulations of $\Omega_{I P}$ are given in ${ }^{18,19}$. The $\Omega$ 's depend on the atomic number, Z, of the nucleus and the transition energy, $\Delta E$. The variation of $\Omega(Z, \Delta E)$ with $\mathrm{Z}$ and $\Delta E$ is moderate, e.g., $\Omega_{K}(\mathrm{Z}=40,1 \mathrm{MeV}) / \Omega_{K}(\mathrm{Z}=80,1 \mathrm{MeV})=2 \times 10^{-3}$, 
$\Omega_{K}(\mathrm{Z}=80,100 \mathrm{keV}) / \Omega_{K}(\mathrm{Z}=80,1 \mathrm{MeV})=0.28$. For $\mathrm{Z}=80, \Delta E=500 \mathrm{keV}$, and $\rho^{2} \simeq 1 \times 10^{-3}$, one obtains $\tau(\mathrm{E} 0) \simeq 3 \mathrm{~ns}$, i.e., $T_{1 / 2}(\mathrm{E} 0) \simeq 2$ ns. Values of $\rho^{2}$ are generally $10^{-1} \sim 10^{-3}$ and so it is standard practice to quote $\rho^{2} \cdot 10^{3}$. The nuclear structure information is contained in $\rho^{2}$.

The nuclear structure information embodied in $\rho^{2}$ values can be directly related to simple models. The monopole operator, cf. Equations (1) and (3), is directly connected to nuclear mean-square charge radii. We present a few details of the electric monopole operator expressed in terms of the shell model and simple geometric and algebraic collective models.

\section{B. The Shell Model}

Shell model estimates of $\rho^{2}(\mathrm{E} 0)$, Equation (3), can be directly formulated because the shell model describes the nucleus in terms of nucleon degrees of freedom. Consider $n$ nucleons confined to a single oscillator shell, $N$. Then, expressing the initial and final states as

$$
\left|\psi_{i}\right\rangle=\sum_{m} a_{m}^{i}\left|\psi_{m}\right\rangle \quad, \quad\left|\psi_{f}\right\rangle=\sum_{l} a_{l}^{f}\left|\psi_{l}\right\rangle
$$

where

$$
\left\{\left|\psi_{m}\right\rangle\right\}=\left\{\left|\left(j_{1}\right)^{n_{1}}\left(j_{2}\right)^{n_{2}} \cdots\left(j_{k}\right)^{n_{k}} ; J M\right\rangle, n=n_{1}+n_{2}+\cdots+n_{k}\right\} \quad,
$$

and $j_{1}, j_{2} \cdots, j_{k}$ are the orbitals making up the shell, we obtain

$$
\rho_{f_{i}}^{2}=\left|\sum_{m} \sum_{l} a_{m}^{i} a_{l}^{f *}\left\langle\psi_{l}\left|\sum_{k=1}^{n} e_{k} r_{k}^{2}\right| \psi_{m}\right\rangle\right|^{2} \frac{1}{e^{2} R^{4}}
$$

The scalar nature of $r_{k}^{2}$ results in $l=m$. Thus,

$$
\rho_{f_{i}}^{2}=\left|\sum_{l} a_{l}^{i} a_{l}^{f *} \sum_{k=1}^{n} e_{k}\left\langle\psi_{l}\left|r_{k}^{2}\right| \psi_{l}\right\rangle\right|^{2} \frac{1}{e^{2} R^{4}}
$$

Because the matrix elemetns of $r_{k}^{2}$ within a single harmonic oscillator shell are independent of $l$ and because of orthogonality of the initial and final states, i.e.,

$$
\sum_{l} a_{l}^{i} a_{l}^{f *}=0
$$

one immediately obtains the result of vanishing $\rho_{f_{i}}^{2}$ values for nucleons confined to a single oscillator shell.

Although $\rho_{f_{i}}^{2}$ vanishes within a single oscillator shell, nucleons in a nucleus do not occupy energy eigenstates which are harmonic oscillator eigenstates. Within an average single-particle field description, nucleons are well described by eigenstates of a Woods-Saxon potential which differs from an oscillator potential by being finite in depth and more "square" in radial profile. These eigenstates can be (and usually are) expressed as linear combinations of oscillator eigenstates. Although the linear combinations are dominated by oscillator eigenstates of a particular shell, there are admixtures of eigenstates from other shells.

A simple expression for $\rho_{f_{i}}^{2}$, when oscillator shell mixing is present, can be directly obtained by considering two states

$$
\begin{aligned}
& \left|0_{i}^{+}\right\rangle=\alpha\left|j_{1}^{2}, 0^{+}\right\rangle+\beta\left|j_{2}^{2}, 0^{+}\right\rangle, \\
& \left|0_{f}^{+}\right\rangle=-\beta\left|j_{1}^{2}, 0^{+}\right\rangle+\alpha\left|j_{2}^{2}, 0^{+}\right\rangle,
\end{aligned}
$$

where $\left|j_{1}^{2}, 0^{+}\right\rangle$and $\left|j_{2}^{2}, 0^{+}\right\rangle$denote pairs of nucleons coupled to total spin zero in oscillator configurations $j_{1}$ and $j_{2}$. Then

$$
\begin{aligned}
\left\langle 0_{f}^{+}\left|r^{2}\right| 0_{i}^{+}\right\rangle= & \left(\alpha^{2}-\beta^{2}\right)\left\langle j_{1}^{2}, 0^{+}\left|r^{2}\right| j_{2}^{2}, 0^{+}\right\rangle \\
& +\alpha \beta\left(\left\langle j_{2}^{2}, 0^{+}\left|r^{2}\right| j_{2}^{2}, 0^{+}\right\rangle-\left\langle j_{1}^{2}, 0^{+}\left|r^{2}\right| j_{1}^{2}, 0^{+}\right\rangle\right)
\end{aligned}
$$

If $j_{1}$ and $j_{2}$ belong to different oscillator shells, then the " $\alpha \beta$ " term depends on the difference in meansquare radii of two oscillator shells. In the limit of maximal mixing, $\alpha=\beta=\frac{1}{\sqrt{2}}$, for $j$ 's in adjacent oscillator shells, 


$$
\left\langle 0_{f}^{+}\left|r^{2}\right| 0_{i}^{+}\right\rangle=\frac{\hbar}{m \omega},
$$

where $\omega$ is the oscillator constant and $m$ is the mass of the nucleon, whence

$$
\rho_{f_{i}}^{2}=0.5 A^{-2 / 3} \quad,
$$

where $\mathrm{A}$ is the nuclear mass number. This gives, for example, $\rho^{2} \cdot 10^{3}$ values of 43 and 15 for $\mathrm{A}=40$ and 200 , respectively. Equation (14) also provides a useful scaling of $\rho^{2}$ in terms of A, i.e., $\rho^{2} \cdot \mathrm{A}^{2 / 3}$ should be essentially independent of mass effects.

An additional and rather subtle point needs to be mentioned. Because nucleons are bound in a finite potential well, the less bound a nucleon, the larger its mean-square radius. Hence, even in a single shell, there are variations in $\left\langle j\left|r^{2}\right| j\right\rangle$. This is well illustrated in the survey of Moalem ${ }^{20}$. We note in particular that although spin-orbit intruder states come from the next higher oscillator shell, their mean-square radii are only slightly larger than valence oscillator shell values. Thus, in practice, Equation (14) is of little practical value, except as a guide to the A dependence of $\rho^{2}$.

Finally, we make a remark about contributions of neutrons to E0 transition strength. One would expect neutrons to make no contribution; however neutrons interact strongly with protons and polarize the nuclear core. Thus, even in nuclei such as ${ }_{28}^{58} \mathrm{Ni}_{30}$ and ${ }_{82}^{206} \mathrm{~Pb}_{124}$ (which we will specifically consider in Section $3 \mathrm{~A}$ ) one finds E0 transition strength.

\section{Geometrical Collective Models}

Geometrical collective models, i.e., models that depend on shape degrees of freedom, as introduced by Bohr and Mottelson ${ }^{1}$ and by the Frankfurt group ${ }^{2,21,22}$, can provide estimates of $\rho^{2}(\mathrm{E} 0)$. There are two important considerations : connecting the collective variables to the monopole operator ; and selection rules. We consider the harmonic quadrupole vibrator and the quadrupole deformed rotor.

\section{The Harmonic Quadrupole Vibrator}

The harmonic quadrupole vibrator describes the nucleus as a sphere with a sharply-defined surface which can execute quadrupole shape oscillations, i.e.,

$$
R(\theta, \phi)=R_{0}\left\{1+\alpha_{00} Y_{00}+\sum_{\mu} \alpha_{\mu} Y_{2 \mu}^{*}(\theta, \phi)\right\} \quad
$$

where $\alpha_{00} Y_{00}$ is included to ensure volume conservation and the $\alpha_{\mu}, \mu= \pm 2, \pm 1,0$ are time-dependent shape parameters. Starting from the general definition of the monopole moment, as described in the Bohr-Mottelson model ${ }^{1}$,

$$
m(E 0)=\int \rho(\vec{r}) r^{2} d \vec{r}
$$

where a continuous charge distribution is assumed*, one obtains

$$
\hat{T}(E 0)=\frac{3}{5} Z e R_{0}^{2}\left(1+\frac{5}{4 \pi} \sum_{\mu}\left|\alpha_{\mu}\right|^{2}\right) \quad,
$$

with only the $\left|\alpha_{\mu}\right|^{2}$ part causing E0 transitions.

Phonon creation and annihilation operators can be introduced

$$
\alpha_{\mu}=\left(\frac{\hbar \omega}{2 C}\right)^{1 / 2}\left(b_{\mu}+(-1)^{\mu} b_{\mu}^{+}\right),
$$

where $C$ is the restoring force constant for harmonic quadrupole vibrations of the nucleus. From the quadratic dependence of $\hat{T}(\mathrm{E} 0)$ on $\alpha_{\mu}$, Equation (17), the selection rules for E0 transitions are

${ }^{*}$ For a density corresponding to point-like charged particles, as in the shell-model, this definition reduces to Equation (1). 


$$
\Delta N=0, \pm 2 \quad
$$

where $N$ is the phonon number. Further, designating the vibrational eigenstates by $|N, R\rangle$, where $R$ labels the collective angular momentum, then

$$
\langle N, R|\hat{T}(E 0)| N, R\rangle=\left(N+\frac{5}{2}\right) \frac{3}{4 \pi} Z e R_{0}^{2} \cdot \frac{\hbar \omega}{C} \quad,
$$

and

$$
\begin{aligned}
\langle N, R|\hat{T}(E 0)| N-2, R\rangle= & \sum_{R^{\prime}}\left\langle N, R\left\|b^{\dagger}\right\| N-1, R^{\prime}\right\rangle\left\langle N-1, R^{\prime}\left\|b^{\dagger}\right\| N-2, R\right\rangle \\
& \times(-1)^{R+R^{\prime}}\left\{\frac{1}{2 \sqrt{2 R+1}}\right\} \frac{3}{4 \pi} Z e R_{0}^{2} \frac{\hbar \omega}{C} .
\end{aligned}
$$

This most general expression for E0 transitions between the collective quadrupole vibrational excitations reduces, e.g., for the $0_{2}^{+} \rightarrow 0_{1}^{+}$E0 transition, to the result

$$
\langle 2,0|\hat{T}(E 0)| 0,0\rangle=\sqrt{\frac{5}{2}} \frac{\hbar \omega}{C} \frac{3}{4 \pi} Z e R_{0}^{2} \quad .
$$

Using the mean-square value

$$
\beta_{r m s}^{2} \equiv\left\langle 0,\left.0\left|\sum\right| \alpha_{\mu}\right|^{2} \mid 0,0\right\rangle=\frac{5}{2} \frac{\hbar \omega}{C}
$$

this equation can be cast in the useful form,

$$
\langle 2,0|\hat{T}(E 0)| 0,0\rangle=\sqrt{\frac{2}{5}} \beta_{r m s}^{2} \frac{3}{4 \pi} Z e R_{0}^{2}
$$

We should point out here that the use of the $B$ and $C$ (or $\omega$ ) values, as obtained from a model with irrotational flow, do not result in the correct magnitudes for collective properties (i.e., $E_{x}\left(2_{1}^{+}\right)$and $\mathrm{B}(\mathrm{E} 2)$ values are not reproduced). For consistency, we shall resort to the use of values of $C, B$ (or $\omega$ ) and derived quantitites from the experimental $E_{x}\left(2_{1}^{+}\right), B\left(E 2 ; 2_{1}^{+} \rightarrow 0_{1}^{+}\right)$values and equate these to the harmonic results. Thus, it is possible to rewrite the value $\rho_{21}^{2}$, using only known quantities, as

$$
\rho_{21}^{2}=\frac{2}{5} \frac{B\left(E 2 ; 0_{1}^{+} \rightarrow 2_{1}^{+}\right)^{2}}{\left(\frac{3}{4 \pi}\right)^{2} Z^{2} r_{0}^{8} A^{8 / 3}}
$$

with $R_{0}=r_{0} A^{1 / 3}, r_{0}=1.2 \mathrm{fm}$ and $\mathrm{B}(\mathrm{E} 2)$ in units of $\mathrm{e}^{2} \cdot \mathrm{fm}^{4}$.

\section{The Quadrupole Deformed Rotor}

The quadrupole deformed rotor describes the nucleus as a deformed body whose surface is sharply defined and given by Equation (15), where some or all of the $\alpha_{\mu}$ 's have non-zero time-independent as well as time-dependent parts. The latter correspond to vibrational oscillations of the deformed equilibrium shape $^{1,2}$. Following the standard transformation ${ }^{\dagger 1,2}$ to a set of body-fixed principal axes, $\left\{\alpha_{\mu}\right\} \rightarrow a_{0}, a_{2} \equiv$ $\left\{\beta_{0}+\xi, \eta\right\}$, where $\left\{\beta_{0}, 0\right\}$ corresponds to the equilibrium shape and $\{\xi, \eta\}$ corresponds to the shape oscillation, one obtains via Equation (17)

$$
\hat{T}(E 0)=\frac{3 Z e R_{0}^{2}}{4 \pi}\left(\beta_{0}^{2}+2 \xi \beta_{0}+\xi^{2}+2 \eta^{2}\right) \quad .
$$

The first term corresponds to the equilibrium shape and will only contribute to E0 moments, whence for E0 transitions,

$$
\hat{T}(E 0)=\frac{3 Z e R_{0}^{2}}{2 \pi}\left(\xi \beta_{0}\right)
$$

\footnotetext{
${ }^{\dagger}$ An alternative parametrization is $\{\beta, \gamma\}$ with $a_{0}=\beta \cos \gamma$ and $a_{2}=\frac{1}{\sqrt{2}} \beta \sin \gamma$
} 
where higher-order contributions of the vibrational oscillation are neglected.

Equation (27) gives rise to non-vanishing E0 transitions deexciting the $\beta$-vibrational excitation (characterized by $n_{\beta}=1$ ) but results in vanishing gamma-band to ground-band E0 matrix elements. The result ${ }^{23}$ is

$$
\rho^{2}\left(E 0 ; n_{\beta}=1 \rightarrow n_{\beta}=0\right)=\frac{1}{2}\left(\frac{3}{2 \pi}\right)^{2} Z^{2} \beta_{0}^{2} \frac{\hbar \omega}{C}
$$

Note that this expression is exact up to second order.

The remark made in Section $2 \mathrm{C} 1$ regarding the use of the $B$ and $C$ coefficients applies here also. We make use of the simple axial rotor expressions but with the parameters adjusted to reproduce the experimental values. Using the definition of the moment of inertia of the ground band, $J_{0}=3 B \beta_{0}^{2}$, $E\left(2_{1}^{+}\right)=\frac{3 \hbar^{2}}{J_{0}}, E\left(0_{\beta}^{+}\right)=\hbar \omega$, alternative expressions using the $\mathrm{E} 2$ transition probability can be derived as

$$
\rho^{2}\left(E 0 ; n_{\beta}=1 \rightarrow n_{\beta}=0\right)=\frac{9}{8 \pi^{2}} Z^{2} \beta_{0}^{4} \frac{E\left(2_{1}^{+}\right)}{E\left(0_{\beta}^{+}\right)}=\frac{B\left(E 2 ; 0_{g}^{+} \rightarrow 2_{\beta}^{+}\right) 4 \beta_{0}^{2}}{e^{2} r_{0}^{4} A^{4 / 3}},
$$

(with $r_{0}=1.2 \mathrm{fm}, \mathrm{B}(\mathrm{E} 2)$ in units of $\mathrm{fm}^{4}$, and $\beta_{0}$ the quadrupole equilibrium deformation). We also point out that $\mathrm{E} 0$ transition rates from the $\beta$ band to the $\mathrm{g}$ band do not contain any angular momentum dependence, i.e., $0^{+} \rightarrow 0^{+}, 2^{+} \rightarrow 2^{+}, 4^{+} \rightarrow 4^{+}, \ldots$ E0 rates are all identical.

\section{Algebraic Collective Models}

Algebraic collective models, i.e., models that are expressed only in terms of creation and annihilation operators which describe collective quanta, can provide a semi-empirical framework for discussing $\rho^{2}$ (E0) values. Further, if simple dynamical symmetries are assumed to be present in the collective structure of nuclei, some selection rules arise. The most extensively developed of the algebraic collective models is the interacting boson model (IBM) which is formulated in two versions : IBM-1 which does not distinguish between protons and neutrons and IBM-2 which does distinguish between them ${ }^{24-27}$.

\section{The Interacting Boson Model - IBM-1}

The IBM-1 provides an explicit language for expressing Hamiltonians and other operators in terms of $s(L=0)$ and $d(L=2)$ bosons $^{27}$. The E0 operator can be written directly as

$$
\hat{T}(E 0)=\beta_{0}\left(d^{\dagger} \tilde{d}\right)^{(0)}+\gamma_{0}\left(s^{\dagger} s\right)^{(0)},
$$

where $\beta_{0}$ and $\gamma_{0}$ are free parameters (not to be confused with the parameters used in Section 2C1) and the superscript notation indicates spherical tensor coupling. Equation (30) can be expressed in terms of the boson number operators $\hat{n}_{s}, \hat{n}_{d}$ and $\hat{N}\left(=\hat{n}_{s}+\hat{n}_{d}\right)$ as

$$
\hat{T}(E 0)=\beta_{0}^{\prime} \hat{n}_{d}+\gamma_{0} \hat{n}_{s}=\gamma_{0} \hat{N}+\tilde{\beta}_{0} \hat{n}_{d}=\beta_{0}^{\prime} \hat{N}+\tilde{\gamma}_{0} \hat{n}_{s} \quad,
$$

where

$$
\beta_{0}^{\prime}=\frac{\beta_{0}}{\sqrt{5}} \quad, \quad \tilde{\beta}_{0}=\beta_{0}^{\prime}-\gamma_{0} \quad, \quad \tilde{\gamma}_{0}=\gamma_{0}-\beta_{0}^{\prime} \quad .
$$

The IBM-1 possesses simple limiting dynamical symmetries which lead to closed form expressions for the matrix elements of $\hat{T}(\mathrm{E} 0)$ and, consequently, to selection rules ${ }^{2-26}$. We deal with the three limiting cases, $\mathrm{U}(5), \mathrm{SU}(3)$, and $\mathrm{O}(6)$, separately.

The U(5) limit of the IBM-1 possesses $N$ and $n_{d}$ as good quantum numbers ${ }^{24}$. Thus, $\hat{T}(\mathrm{E} 0)$ is diagonal in this limit and $\mathrm{E} 0$ transitions are forbidden.

The SU(3) limit of the IBM-1 possesses $N$ as a good quantum number together with the conventional $\mathrm{SU}(3)$ quantum numbers $(\lambda, \mu)$, but $n_{d}$ is not a good quantum number ${ }^{25}$. However, considering $\hat{T}(\mathrm{E} 0)$ in the form

$$
\hat{T}(E 0)=\gamma_{0} \hat{N}+\tilde{\beta}_{0} \sqrt{5}\left(d^{\dagger} d\right)^{(0)},
$$

noting that $d^{\dagger}$ transforms ${ }^{27}$ as a $(\lambda, \mu)=(2,0)$ representation of $\mathrm{SU}(3)$; then, e.g., $(\lambda, \mu)=(2 N, 0)$ states connect only with $(\lambda, \mu)=(2 N-4,2)$ states via E0 transitions. This includes the E0 transitions 
connecting the " $\beta$ " band $((2 N-4,2)$ irrep. $)$ with the g band $((2 N, 0)$ irrep. $)$. The E0 matrix element becomes

$$
\begin{aligned}
& \langle(2 N, 0) K=0, L|\hat{T}(E 0)|(2 N-4,2) K=0, L\rangle \\
& =\tilde{\gamma}_{0} N\left[\frac{(2 N-L)(2 N+L+1)}{3(2 N-1)(2 N)}\right]^{1 / 2}\left[\frac{2(2 N-1)^{2}-L(L-1)}{3(2 N-2)(2 N-1)}\right]^{1 / 2}\left[\frac{2(N-1)}{N(2 N-3)}\right]^{1 / 2}
\end{aligned}
$$

This simplifies

(i) for $N \rightarrow \infty$ to

$$
\widetilde{\gamma}_{0} \frac{\sqrt{2 N}}{3}
$$

(ii) for $L=0$ to

$$
\widetilde{\gamma}_{0} \sqrt{\frac{2 N(2 N+1)}{9(2 N-3)}}
$$

(iii) for the ratio $R$ to :

$$
R \equiv \frac{M(E 0 ; L \rightarrow L)}{M(E 0 ; 0 \rightarrow 0)}=\left[\frac{(2 N-L)(2 N+L+1)}{2(2 N)(2 N+1)} \cdot \frac{2(2 N-1)^{2}-L(L+1)}{(2 N-1)^{2}}\right]^{1 / 2}
$$

and thus expresses a specific $L$ dependence in the $\beta$ band $\rightarrow \mathrm{g}$ band E0 transitions. This is illustrated in Figure 1.

The $\mathrm{O}(6)$ limit of the IBM-1 possesses $N$ as a good quantum number together with the conventional $\mathrm{O}(6)$ quantum numbers $\sigma, \tau$ but $n_{d}$ is not a good quantum number ${ }^{26}$. The $\mathrm{E} 0$ transition operator possesses the selection rules $\Delta \sigma=0, \pm 2 ; \Delta \tau=0$. Thus, the E0 matrix elements that connect to the $0^{+}$ground-state level $|[N], \sigma=N, \tau=0, L=0\rangle$ originate in the $\sigma=N-2$ multiplet, i.e.. $|[N], \sigma=N-2, \tau=0, L=0\rangle$

$$
\begin{aligned}
\langle[N], \sigma=N, \tau=0, L=0|\hat{T}(E 0)|[N], \sigma & =N-2, \tau=0, L=0\rangle \\
& =\tilde{\beta}_{0} \frac{\sqrt{(N-2)(N-1)(N+3)}}{2(N+1)},
\end{aligned}
$$

which simplifies, for large $N(N \rightarrow \infty)$, to the result

$$
M(E 0)_{\sigma=N-2 \rightarrow \sigma=N} \cong \tilde{\beta}_{0} \frac{\sqrt{N}}{2} .
$$

The forbiddenness of E0 transitions in the U(5) limit of IBM-1 and their allowed character in the harmonic quadrupole vibrator needs some comment. Primarily, the model operators are quite different (cf. Equations (17) and (31)). For the geometric model, the $\Delta \mathrm{N}=0, \pm 2$ selection rules (Equation (19)) follow directly. For the boson model, the operator is the simplest monopole operator that can be constructed from the boson operators. It is fair to say that the boson model E0 operator is too simplistic. There are other concerns with the interacting boson model : the bosons of the model are regarded as superpositions of pair-correlated configurations restricted to the valence shell. This has been formalized in the OAI mapping procedure ${ }^{28}$. Restriction to a valence shell within a harmonic oscillator-based shell model, as noted earlier, would result in vanishing E0 matrix elements. Thus, we infer that the description of E0 transitions within the IBM-1 is probably seriously deficient.

\section{The Interacting Boson Model - IBM-2}

The IBM-2 provides a more detailed language than IBM-1 for the description of collectivity in nuclei in that it distinguishes between protons and neutrons. Thus, the E0 operator can be written (cf. Equation (31))

$$
\hat{T}(E 0)=\gamma_{0 \pi} \hat{N}_{\pi}+\tilde{\beta}_{0 \pi} \hat{n}_{d \pi}+\gamma_{0 \nu} \hat{N}_{\nu}+\tilde{\beta}_{0 \nu} \hat{n}_{d \nu}
$$


The IBM-2 possesses simple limiting dynamical symmetries similar to IBM-1 and in addition new types of limiting symmetries that depend on the distinction between protons and neutrons. The formalism is extensive and is fully detailed in a monograph ${ }^{29}$. A discussion of some aspects of E0 transitions within an IBM-2 framework is made by Barrett and Otsuka ${ }^{30}$. Most commonly, the application of IBM-2 is via parameter fitting to systematic trends in long chains of isotopes (or isotones) with only a few parameters varying. Specific papers using this latter approach will, where applied to E0 properties of nuclei, be cited in Section 3.

We reiterate here, the concern expressed at the end of the previous section, that the description of E0 transitions in IBM-2 may also be seriously deficient. Additional caution is needed in using Equation (40), cf. Equation (31), because there are more fitting parameters available.

\section{E. Shape Mixing Effects on E0 Transitions}

The connection between shape mixing and E0 transitions is the essential point that we want to make in this paper. We argue that, in general, nuclei characterized by coexisting shapes having different deformations will exhibit strong $\rho^{2}(\mathrm{E} 0)$ values if the states associated with the coexisting shapes become mixed. We present this argument starting from a two-level mixing description.

Simple two-level models consist of two configurations corresponding to, in general, two shapes with different quadrupole equilibrium values in the appropriate deformation $(\beta)$ space. In the general case, mixing between these two configurations $\left|0_{1}^{+}\right\rangle$and $\left|0_{2}^{+}\right\rangle$will result in two eigenstates $\left|0_{i}^{+}\right\rangle$and $\left|0_{f}^{+}\right\rangle$, respectively (see Figure 2)

$$
\begin{aligned}
& \left|0_{i}^{+}\right\rangle=a\left|0_{1}^{+}\right\rangle+b\left|0_{2}^{+}\right\rangle, \\
& \left|0_{f}^{+}\right\rangle=-b\left|0_{1}^{+}\right\rangle+a\left|0_{2}^{+}\right\rangle,
\end{aligned} .
$$

Using a general E0 operator, $\hat{T}(\mathrm{E} 0)$, which needs to be expressed in the appropriate model space, characterized by a single quadrupole deformation variable $\beta$, one obtains the result for $\rho_{f i}(\mathrm{E} 0)$

$$
\begin{aligned}
\rho_{f i}(E 0)= & \frac{1}{e R^{2}}\left[a b\left(\left\langle 0_{1}^{+}|\hat{T}(E 0)| 0_{1}^{+}\right\rangle-\left\langle 0_{2}^{+}|\hat{T}(E 0)| 0_{2}^{+}\right\rangle\right)\right. \\
& \left.+\left(a^{2}-b^{2}\right)\left\langle 0_{2}^{+}|\hat{T}(E 0)| 0_{1}^{+}\right\rangle\right]
\end{aligned}
$$

First, consider the situation in which weak mixing occurs between configurations that correspond to strongly differing equilibrium shapes. Thus,

$$
a b \simeq 0 \quad,
$$

because of weak mixing; and

$$
\left\langle 0_{2}^{+}|\hat{T}(E 0)| 0_{1}^{+}\right\rangle \simeq 0
$$

because the wave functions are mainly localized at different points in deformation space. Thus, $\rho_{f i}^{2} \simeq 0$. The best example, shown in Figure 3, is observed for the E0 decay from the fission isomeric $0^{+}$state in ${ }^{238} \mathrm{U}^{31,32}$, resulting in the most retarded E0 transition observed anywhere (cf. Table 3 ).

Second, consider the strong mixing situation. We still have $\left\langle 0_{2}^{+}|\hat{T}(E 0)| 0_{1}^{+}\right\rangle \simeq 0$, but

$$
a \simeq-b \simeq \frac{1}{\sqrt{2}}
$$

Thus, the general result (42) is approximated by

$$
\rho(E 0)=\frac{1}{2}\left[\left\langle 0_{1}^{+}|\hat{T}(E 0)| 0_{1}^{+}\right\rangle-\left\langle 0_{2}^{+}|\hat{T}(E 0)| 0_{2}^{+}\right\rangle\right] \frac{1}{e R^{2}} \quad .
$$

Here, use can be made of the fact that in the collective quadrupole deformation space we have the definition

$$
\beta_{k}^{2} \equiv\left\langle\left. 0_{k}^{+}\left|\sum\right| \alpha_{\mu}\right|^{2} \mid 0_{k}^{+}\right\rangle
$$

which leads to the expression 


$$
\rho_{f i}^{2}(E 0)=\frac{1}{4}\left(\frac{3}{4 \pi}\right)^{2} Z^{2}\left(\beta_{1}^{2}-\beta_{2}^{2}\right)^{2}
$$

In the particular situation where we have strong mixing between an almost spherical and a strongly deformed shape, we obtain the result (see also Figure 4)

$$
\rho_{f i}^{2}(E 0) \simeq \frac{1}{4}\left(\frac{3}{4 \pi}\right)^{2} Z^{2} \beta_{d e f}^{4}
$$

\section{F. The Connection Between E0 Transitions and Nuclear Radii}

The connection between E0 transitions and nuclear radii which we wish to make is that there are a number of regions where E0 transitions are observed and where empirical data on $\Delta\left\langle r^{2}\right\rangle$ are now available ${ }^{33}$. Here we present some simple perspectives on trends in $\left\langle r^{2}\right\rangle$ and show how they are connected to nuclear structure. Specifically, we show the types of change in isotope and isomer shifts associated with intruder states $^{\ddagger}$ and sudden changes in ground-state structure and the corresponding behavior expected for $\rho^{2}(\mathrm{E} 0)$.

Starting from a quadrupole (axial) deformed nuclear shape, expressed with a simplified version of Equation (15), i.e.,

$$
R=R_{0}\left(1+\beta Y_{2}^{0}(\theta)\right) \quad,
$$

(with $R_{0}=r_{0} \cdot A^{1 / 3} \mathrm{fm}$ ), one derives the mean-square radius as

$$
\left\langle r^{2}\right\rangle=\frac{3}{5} R_{0}^{2}\left(1+\frac{5}{4 \pi} \beta^{2}\right)
$$

If we now study the situation of two basic configurations (Section $2 \mathrm{E}$ ), corresponding to different equilibrium quadrupole deformation values $\Delta\left(\beta^{2}\right)$, and allow mixing (described by the mixing amplitude $a$ ), the connecting E0 transition is described by (see also ${ }^{34}$ )

$$
\rho^{2}(E 0)=\left(\frac{3}{4 \pi} Z\right)^{2} a^{2}\left(1-a^{2}\right)\left[\Delta\left(\beta^{2}\right)\right]^{2}
$$

The nuclear radius variation between these two configurations is obtained from Equation (50) as

$$
\Delta\left\langle r^{2}\right\rangle=\frac{3}{4 \pi} R_{0}^{2} \Delta\left(\beta^{2}\right) \quad ;
$$

or, connecting the isotope shift to a $\rho^{2}(\mathrm{E} 0)$ value results in the formula

$$
\rho^{2}(E 0)=\frac{Z^{2}}{R_{0}^{4}} a^{2}\left(1-a^{2}\right)\left[\Delta\left\langle r^{2}\right\rangle\right]^{2}
$$

Strictly speaking, the $\Delta\left\langle r^{2}\right\rangle$ value in Equation (52) corresponds to the isomeric shift between the two $0^{+}$ configurations. However, we can argue that this isomeric shift will approximately be equal to the isotopic shift where the two $0^{+}$configurations cross (see below). So, it is a very reasonable assumption to use Equation (53) also as an expression relating the $\rho^{2}(\mathrm{E} 0)$ value to the isotopic shift in those nuclei where strong variations in the nuclear radius are seen.

An interesting consistency test of Equation (53) can be carried out for the nucleus ${ }^{96} \mathrm{Sr}$ because : (i) for a long series of Sr nuclei, the isotope shifts are known experimentally as shown in Figure 5 and, (ii) a $\rho^{2}(\mathrm{E} 0)$ value, one of the largest, is known in ${ }^{96} \mathrm{Sr}$ (cf. Table 1 and Figure 16). If we make use of Equation (53), and make as input choice for the mixing amplitude the maximal value $a=1 / \sqrt{2}$, a theoretical value $\rho^{2}(\mathrm{E} 0)=0.143$ is deduced, in good agreement with the experimental value of $\rho^{2}(\mathrm{E} 0)=0.185 \pm 0.050$. This test shows the validity and usefulness of the relation (53).

\footnotetext{
${ }^{\ddagger}$ In the present discussion, we refer to intruder states as those low-lying excited states that are connected to a largely different deformation as compared to the ground-state deformation. In a microscopic context, they are associated with particle-hole excitations across shell gaps.
} 
The relation between mixing a deformed and a spherical configuration, the variation in nuclear radius in the ground state (isotopic shift), and $\rho^{2}$ (E0) values connecting the two $0^{+}$levels can be highlighted in a simple two-state (mixing) model. In the case of no mixing, sharp $\left\langle r^{2}\right\rangle$ variations (spikes) and vanishing $\rho^{2}(\mathrm{E} 0)$ values will be seen. With increasing mixing between the two $0^{+}$states, the $\left\langle r^{2}\right\rangle$ variation will become more gradual and $\rho^{2}(\mathrm{E} 0)$ strength will appear in the vicinity of the crossing points. This is illustrated in Figures 6 and 7.

The relationship between E0 transition rates and the variation of the nuclear radius can be extended further. In the situation where the ground state contains some admixture of a strongly-deformed configuration, the E0 transition rate can give information on the mixing amplitude. If now the value $\beta$ of the deformed configuration is known (from $\mathrm{B}(\mathrm{E} 2)$ data as in, e.g., the $\mathrm{Hg}, \mathrm{Pt}$ and $\mathrm{Pb}$ nuclei), the mixing amplitude $a$, describing the mixed wave functions

$$
\begin{gathered}
\left|0_{g}^{+}\right\rangle=a\left|0_{\text {sph. }}^{+}\right\rangle+\sqrt{1-a^{2}}\left|0_{d e f}^{+}\right\rangle \\
\left|0_{\text {exc. }}^{+}\right\rangle=-\sqrt{1-a^{2}}\left|0_{s p h .}^{+}\right\rangle+a\left|0_{d e f}^{+}\right\rangle,
\end{gathered}
$$

can be deduced from Equation (51).

\section{Manifestation of E0 Transitions in Nuclei}

In this section we undertake a detailed classification of experimental $\rho^{2}(\mathrm{E} 0)$ values in terms of the different types of structure observed in nuclei, i.e., shell model, collective models, and shape-coexistence models. This is the essential part of the present paper. The subsections - singly-closed shell (and subshell) nuclei, vibrational and weakly-deformed open-shell nuclei, strongly-deformed nuclei, nuclei that exhibit a sudden change in ground-state structure, nuclei that exhibit shape coexistence and intruder states, light nuclei $(\mathrm{A}<56)$, and odd-A nuclei - are chosen to classify both nuclei of well-defined structural types (cf. the preceeding section) and nuclei which exhibit large $\rho^{2}(\mathrm{E} 0)$ values.

There are surprisingly few $\rho^{2}(\mathrm{E} 0)$ values known. We present all known values in Tables 1-4. Many more E0 transitions have been identified in nuclei; however a lack of half-life data precludes the assignment of $\rho^{2}(\mathrm{E} 0)$ to these transitions. We emphasize here that this is a serious experimental deficiency in our knowledge of nuclei. We do not undertake a compilation of E0 transitions for which there are no half-life data available. Nevertheless, where patterns of E0 decay help to understand $\rho^{2}(\mathrm{E} 0)$ information, we present details. In the various subsections we reiterate the data, usually in figures. Finally, we note the use of $\rho^{2} \cdot A^{2 / 3}$ (cf. Table 4 ) which provides a mass-independent comparison of values.

\section{A. Singly-Closed Shell Nuclei}

In this section we present data for singly-closed shell nuclei with $\mathrm{A} \geq 56$ and the zirconium nuclei with $\mathrm{N} \leq 56$ which have a closed subshell. However, we postpone a discussion of the (singly-closed shell) tin and lead nuclei, which lie near to the respective midshells at $\mathrm{N}=66$ and 104, until Section $3 \mathrm{E}$ where we deal with low-lying intruder states which are important in these nuclei.

Figure 8 shows the $\rho^{2}(\mathrm{E} 0)$ data for the nickel isotopes $(\mathrm{Z}=28)^{36,37}$. Of particular note are the transitions with $\rho^{2}(\mathrm{E} 0) \cdot 10^{3} \sim 80$ units which rank among the strongest known for $\mathrm{A} \geq 56$. In ${ }^{58,60} \mathrm{Ni}$ the parent levels (at 3531 and $3319 \mathrm{keV}$ ) match states which are strongly populated in $\left({ }^{3} \mathrm{He}, \mathrm{n}\right)$ proton-pair transfer reactions ${ }^{38,39}$, i.e., these levels are most probably $\mathrm{Z}=28$ proton intruder states. It appears that in ${ }^{62} \mathrm{Ni}$ the proton intruder configuration resides predominantly in the $3519 \mathrm{keV}$ state $^{40}$, thus raising a question about the $\rho^{2}$ (E0) strength associated with the $2049 \mathrm{keV}$ state. However, there are no direct experimental data on the admixture of the proton intruder configuration in the $2049 \mathrm{keV}$ state. Lastly, ${ }^{58} \mathrm{Ni}$ is remarkable in that it exhibits both one of the strongest and one of the weakest known $\rho^{2}(\mathrm{E} 0)$ values with a difference of $\sim 10^{4}$. We interpret the $2942 \mathrm{keV}$ state in ${ }^{58} \mathrm{Ni}$ as a valence neutron shell configuration.

The appearance of strong $\rho^{2}(\mathrm{E} 0)$ values in the ${ }^{58-62} \mathrm{Ni}$ nuclei implies significant mixing between the proton $2 \mathrm{p}-2 \mathrm{~h}$ intruder configurations and the ground state. This is surprising in the light of the high excitation energy $\left(E_{x} \simeq 3 \mathrm{MeV}\right)$ of the $2 \mathrm{p}$ - $2 \mathrm{~h}$ states. The presence, in ${ }^{58} \mathrm{Ni}$, of one of the weakest $\rho^{2}(\mathrm{E} 0)$ values is easier to understand. The nucleus ${ }^{58} \mathrm{Ni}$ has two neutrons outside of a doubly-closed shell. A variety of $0^{+}$pair configurations can arise within the neutron valence shell. These will all have essentially the same $\left\langle r^{2}\right\rangle$ values. Further, when there are only valence neutrons outside of a doubly-closed shell, in order to produce E0 transition strength, the neutrons must polarize the core and this polarization must be different for different configurations.

Figure 9 shows the $\rho^{2}(\mathrm{E} 0)$ data for the zirconium isotopes $(\mathrm{Z}=40)^{41-48}$. Although $\mathrm{Z}=40$ is not a major closed shell, a proton sub-shell gap exists for ${ }^{90-96} \mathrm{Zr}$. The most notable feature in the figure is the large 
$\rho^{2}(\mathrm{E} 0)$ values for ${ }^{98,100} \mathrm{Zr}$. These are due to a sudden shape change and we defer their discussion until Section $3 \mathrm{D}$.

One can analyze the $\rho^{2}(\mathrm{E} 0)$ values up to $\mathrm{N}=56$ using a two-component shell-model calculation ${ }^{42,43,45}$, in which the $\left(2 p_{1 / 2}\right)_{0^{+}}^{2}$ and $\left(1 g_{9 / 2}\right)_{0^{+}}^{2}$ shell-model configurations dominate the structure of the low-lying $0^{+}$states. The pairing force strongly mixes these configurations, resulting in

$$
\begin{aligned}
& \left|0_{1}^{+}\right\rangle=\alpha\left|\left(2 p_{1 / 2}\right)^{2} 0^{+}\right\rangle+\beta\left|\left(1 g_{9 / 2}\right)^{2} 0^{+}\right\rangle, \\
& \left|0_{2}^{+}\right\rangle=-\beta\left|\left(2 p_{1 / 2}\right)^{2} 0^{+}\right\rangle+\alpha\left|\left(1 g_{9 / 2}\right)^{2} 0^{+}\right\rangle
\end{aligned} .
$$

Spectroscopic factors deduced from transfer reactions (see refs. in ${ }^{43}$ and $^{49}$ ) give $\alpha$ and $\beta$ values. Thus,

$$
\left\langle 0_{1}^{+}|\hat{T}(E 0)| 0_{2}^{+}\right\rangle=2 e \alpha \beta\left\{\left\langle r^{2}\right\rangle_{2 p_{1 / 2}}-\left\langle r^{2}\right\rangle_{1 g_{9 / 2}}\right\}
$$

Figure 10 shows the experimental values for $\rho_{21}=\rho\left(0_{2}^{+} \rightarrow 0_{1}^{+}\right)$for ${ }^{90,92,94,96} \mathrm{Zr}$ and comparison with $\rho_{21}$ values estimated using Equation (56) with $\left(\left\langle r^{2}\right\rangle_{2 p_{1 / 2}}-\left\langle r^{2}\right\rangle_{1 g_{9 / 2}}\right)$ values given by the shell model estimate, i.e., $\mathrm{A}^{1 / 3}$, and by the values $\left\langle r^{2}\right\rangle_{2 p_{1 / 2}}^{1 / 2}=4.64 \mathrm{fm}$ and $\left\langle r^{2}\right\rangle_{1 g_{9 / 2}}^{1 / 2}=4.79 \mathrm{fm}$ determined ${ }^{42}$ using the ${ }^{90} \mathrm{Zr}(\mathrm{t}, \alpha){ }^{89} \mathrm{Y}$ reaction. Evidently, for ${ }^{90} \mathrm{Zr}$, the shell model estimate fails whereas the estimate using empirically-determined $\left\langle r^{2}\right\rangle_{n l j}$ values agrees within experimental error. (We note that the influence of variable occupancy of the $2 \mathrm{p}_{3 / 2}$ orbital has been investigated ${ }^{41-43}$ also.) The zirconium isotopes are discussed further when the sudden shape change between ${ }^{98} \mathrm{Zr}$ and ${ }^{100} \mathrm{Zr}$ is considered.

Figure 11 shows the $\rho^{2}(\mathrm{E} 0)$ data for the $\mathrm{N}=82$ isotones ${ }^{50,51}$. In the $\mathrm{N}=82$ single-closed shell nuclei, protons are filling the $1 g_{7 / 2}, 2 d_{5 / 2}, 2 d_{3 / 2}, 3 s_{1 / 2}$ and $1 h_{11 / 2}$ orbitals. Electric monopole transitions are expected because of mixing between the $\mathrm{N}=4$ orbitals and the $\mathrm{N}=5\left(1 \mathrm{~h}_{11 / 2}\right)$ orbital. The simple oscillator shell estimate of $\rho^{2}$ for ${ }^{142} \mathrm{Nd}$, with 50-50 mixing, is 0.018. Thus, a shell model description would be a good zeroth-order approximation in the $\mathrm{N}=82$ isotones.

The only calculation of E0 properties for the $\mathrm{N}=82$ isotones is a simple two-particle shell model plus pairing force calculation ${ }^{52}$. The calculated E0 properties of ${ }^{134} \mathrm{Te}$ were qualitatively compared with ${ }^{136} \mathrm{Xe}$ E0 transition patterns (no $\rho^{2}$ values are known) and the E0 systematics of the heavier $\mathrm{N}=82$ isotones.

The lowering of the $\rho^{2}$ value in ${ }^{146} \mathrm{Gd}$ compared to ${ }^{142} \mathrm{Nd}$ has been discussed ${ }^{50}$ in terms of a variable effective monopole charge. An alternative possibility is that orbital binding energy effects are playing a role $^{20}$

At the $\mathrm{Z}=82$ closed shell there are $\rho^{2}\left(0_{2}^{+} \rightarrow 0_{1}^{+}\right)$data for ${ }^{206} \mathrm{~Pb}^{53,54}$ and ${ }^{204} \mathrm{~Pb}^{55}$. Tape et al. ${ }^{54}$ have discussed the $\mathrm{E} 0$ decay of the $0_{2}^{+}$state in ${ }^{206} \mathrm{~Pb}$ in terms of simple mixing of valence neutron hole pairs, isotope shift data, and an effective monopole charge for neutrons. They used

$$
\begin{aligned}
& \left|0_{1}^{+}\right\rangle=\alpha\left|\left(3 p_{1 / 2}\right)^{-2} 0^{+}\right\rangle+\beta\left|\left(2 f_{5 / 2}\right)^{-2} 0^{+}\right\rangle \\
& \left|0_{2}^{+}\right\rangle=-\beta\left|\left(3 p_{1 / 2}\right)^{-2} 0^{+}\right\rangle+\alpha\left|\left(2 f_{5 / 2}\right)^{-2} 0^{+}\right\rangle,
\end{aligned}
$$

and obtained $\rho_{t h}=0.016(10)$ which can be compared with $\rho_{\text {expt }}=0.034(2)^{53}$. The same reasoning but now using theoretical isotopic shifts from neutron hole states in ${ }^{207} \mathrm{~Pb}$ relative to ${ }^{208} \mathrm{~Pb}^{56}$, and the theoretical $0_{1}^{+}, 0_{2}^{+}$wave functions determined by $\mathrm{Ma}$ and True ${ }^{57}$, considering the major $3 p_{1 / 2}, 2 f_{5 / 2}$ and $3 p_{3 / 2}$ components results in the value $\rho_{t h}(\mathrm{E} 0)=0.027$.

\section{B. Vibrational and Weakly-Deformed Open-Shell Nuclei}

In this section we present data for nuclei which are the best examples of harmonic quadrupole vibrators. We also look at nuclei which closely match the $\mathrm{U}(5)$ and $\mathrm{O}(6)$ limits of the IBM1 (nuclei which closely match the U(5) limit also closely match harmonic quadrupole vibrations). Lastly, in this section, we consider nuclei which we classify as weakly-deformed open-shell nuclei.

Figure 12 shows $\mathrm{B}(\mathrm{E} 2)$ data for low-lying states in ${ }^{108,110} \mathrm{Pd}^{58,59}$. These two nuclei are very good examples of (near) harmonic quadrupole vibrators ; possibly they are the best known examples. Figure 13 shows the available $\rho^{2}$ (E0) data for the palladium isotopes ${ }^{60-63}$. Evidently, ${ }^{108,110} \mathrm{Pd}$ are not associated with strong $\rho^{2}(E 0)$ values. The nuclei ${ }^{104,106} \mathrm{Pd}$ are also (very) good examples of harmonic quadrupole vibrators (see, e.g., Kern et al. $\left.{ }^{64}\right)$. Likewise, the $\rho^{2}(\mathrm{E} 0)$ values in ${ }^{104,106} \mathrm{Pd}$ are not strong. We infer that a "typical" $\rho^{2}(\mathrm{E} 0) \cdot 10^{3}$ value for the $0_{2} \rightarrow 0_{1}$ transition in these nuclei is $\simeq 4$ units. The vibrational nature of ${ }^{106} \mathrm{Pd}$, which has $\rho^{2}(\mathrm{E} 0) \cdot 10^{3}=14 \pm 5$ units, is not significantly different from its neighbors and we argue that the larger value of $\rho^{2}$ (E0) $\left(0_{2} \rightarrow 0_{1}\right)$ in this nucleus is due either to an intruder admixture ${ }^{5}$ (cf. the neighboring cadmium isotopes discussed in Section $3 \mathrm{E}$ ), or the true value (cf. the experimental 
error) is smaller. These values of $\rho^{2}$ can be compared with an estimate using Equation (25) which gives $\rho^{2}(\mathrm{E} 0) \cdot 10^{3} \approx 200$ for ${ }^{106} \mathrm{Pd}$.

Figure 13 also shows the available $\rho^{2}(\mathrm{E} 0)$ data for the ruthenium isotopes ${ }^{65,66}$. These nuclei have more complex collective structure. The ruthenium isotopes have variously been described using the geometrical collective model (see, e.g. ${ }^{21,22}$ ) and using the interacting boson model (see, e.g., ${ }^{6-69}$ ) : the lighter $\mathrm{Ru}$ isotopes have been interpreted as vibrational or $\mathrm{U}(5)$-like and the heavier $\mathrm{Ru}$ isotopes have been interpreted as axially asymmetric rotors or $\mathrm{O}(6)$-like. Shape coexistence has also been suggested ${ }^{21,22,70}$ in these nuclei. In the context of shape coexistence, it has further been suggested ${ }^{70}$ that enhanced E0 transitions be looked for in ${ }^{104} \mathrm{Ru}$.

Figure 13 shows, lastly, the available $\rho^{2}(\mathrm{E} 0)$ data for the molybdenum isotopes ${ }^{60}$. The large $\rho^{2}(\mathrm{E} 0)$ values in ${ }^{98,100} \mathrm{Mo}$ and the very large value in ${ }^{102} \mathrm{Mo}$ can be explained by shape coexistence and a sudden change in ground-state structure. This is addressed in Section $3 \mathrm{D}$.

Figure 14 shows the available data for the zinc $^{71,72}$, germanium ${ }^{73,74}$, selenium ${ }^{73,75-77}$, and krypton ${ }^{78-81}$ nuclei. The nuclei shown exhibit a rich variety of collective behavior. Most notably, the selenium and krypton isotopes exhibit shape coexistence ${ }^{5}$. Shape coexistence and $\rho^{2}(\mathrm{E} 0)$ values in ${ }^{78} \mathrm{Kr}$ have been discussed $^{82}$ very recently.

The $\mathrm{O}(6)$ limit of the IBM-1 possesses well-defined E0 transition selection rules, cf. Section 2 D 1. The nuclei ${ }^{194,196} \mathrm{Pt}$ are, probably, the closest realization for $\mathrm{O}(6)$ collective behavior known (see, e.g. ${ }^{26,83}$ ) ; but note the cautionary remarks in $\left.{ }^{84}\right)$. Figure 15 shows the available $\rho^{2}(\mathrm{E} 0)$ data for ${ }^{194} \mathrm{Pt}$ (calculated using information in the Nuclear Data Sheets for $\mathrm{A}=194)$ and ${ }^{196} \mathrm{Pt}^{85}$. The $0_{3}^{+} \rightarrow 0_{1}^{+}$transition $(\Delta \sigma=$ $2, \Delta \tau=0)$ is allowed and the $0_{2}^{+} \rightarrow 0_{1}^{+}$transition $(\Delta \sigma=0, \Delta \tau=3)$ and the $0_{3}^{+} \rightarrow 0_{2}^{+}$transition $(\Delta \tau=2, \Delta \tau=3)$ are forbidden. Either the $\mathrm{O}(6) 0^{+}$states in ${ }^{194,196} \mathrm{Pt}$ are incorrectly identified (and possibly intruder states must be invoked ${ }^{85}$ ) or the description of E0 transitions in the IBM-1 (cf. Equation $(30,31)$ ) is wrong. (We note that the E0 transition strength pattern observed in ${ }^{194,196} \mathrm{Pt}$ also does not match predictions made by Barrett and Otsuka ${ }^{30}$ for the $\mathrm{O}(6)$ limit of the IBM-2. Further, the neutrondeficient $\mathrm{Ba}$ and Xe isotopes, which are suggested ${ }^{30}$ as possible examples of the $\mathrm{O}(6)$ limit of the IBM-2, may not obey the predicted monopole decay pattern ${ }^{86}$. Finally, we note some IBM- 2 fits ${ }^{87}$ to monopole properties in the osmium and platinum nuclei which are in fair agreement with the data; and some discussion ${ }^{88}$ of E0 properties of ${ }^{188} \mathrm{Os}$ and ${ }^{196} \mathrm{Pt}$ in terms of IBM-1.)

\section{Strongly-Deformed Nuclei}

Strongly-deformed nuclei are easily identified by their rotational behavior. There have been remarkably few such nuclei for which $\rho^{2}$ (E0) data are available (see, e.g. ${ }^{10,11}$ ). Consequently, we have undertaken a compilation and evaluation of data from which $\rho^{2}(\mathrm{E} 0)$ values have been extracted for deformed nuclei. We present the data in Table 3. (The $\mathrm{N}=90$ nuclei, which exhibit rotational behavior, are discussed in Section $3 \mathrm{D}$ because they lie in a region of sudden change in ground-state structure.)

Traditionally, strongly-deformed nuclei have been discussed in terms of rotations, $\beta$ vibrations, $\gamma$ vibrations, and quasiparticle excitations ; with an association between E0 transitions and $\beta$ vibrations ${ }^{1}$. This association has been based largely on the nuclei ${ }^{152} \mathrm{Sm},{ }^{154} \mathrm{Gd}$, and ${ }^{174} \mathrm{Hf}$. The nuclei ${ }^{152} \mathrm{Sm}$, and ${ }^{154} \mathrm{Gd}$ have $\mathrm{N}=90$ and, as noted above, are discussed in Sect. $3 \mathrm{D}$. The nucleus ${ }^{174} \mathrm{Hf}$ was discussed in detail in ref. ${ }^{1}$ (see ref. ${ }^{1}$, pp. 168-171) ; but the data in Table 3 reveal that ${ }^{174} \mathrm{Hf}$ is only one of a number of nuclei with moderate $\rho^{2}$ (E0) values (moreover, the updated value given in Table 3 is about one half of the original adopted value ${ }^{89}$. This $\beta$-vibrational band picture has probably retained its popularity because E0 transitions are expected with equal strength for all $\Delta J=0, \beta$-band to ground-band transitions (cf. Section $2 \mathrm{C}$ ). Indeed, $\mathrm{J}_{\beta} \rightarrow \mathrm{J}_{g}$ E0 transitions are seen in ${ }^{152} \mathrm{Sm}$ and ${ }^{154} \mathrm{Gd}$ up to $\mathrm{J}=10$ (see Section $3 \mathrm{D}$ ) and in ${ }^{174} \mathrm{Hf}$ up to $\mathrm{J}=8^{889}$. However, the identification of $\beta$ vibrations has generally been elusive and the current picture is confused. We briefly discuss this below.

The answer to the question "Do $\beta$ vibrations exist in nuclei ?" is very unclear. Ideally, $\beta$ vibrations should exhibit $\mathrm{B}\left(\mathrm{E} 2 ; 0_{g}^{+} \rightarrow 2_{\beta}^{+}\right)$values comparable to the $\gamma$-vibrational quantity $\mathrm{B}\left(\mathrm{E} 2 ; 0_{g}^{+} \rightarrow 2_{\gamma}^{+}\right)^{1}$. This is, in general, not observed. The identification of $\gamma$ bands in deformed nuclei has been widely made through their enhanced $\mathrm{B}\left(\mathrm{E} 2 ; 0_{g}^{+} \rightarrow 2_{\gamma}^{+}\right)$values. It is safe to say that $\gamma$ vibrations occur in all deformed nuclei, that the $0_{g}^{+} \rightarrow 2_{\gamma}^{+}$transition has an E2 strength of $2 \sim 10$ single-particle units, and that the $\gamma$-vibrational band is always the lowest $\mathrm{K}^{\pi}=2^{+}$band in a given nucleus. The lowest $\mathrm{K}^{\pi}=0^{+}$bands, which would

${ }^{\S}$ We note that for ${ }^{174} \mathrm{Hf}$, estimates ${ }^{89}$ are made for $\rho^{2}\left(E_{0}, J_{\beta} \rightarrow J_{g}\right)$ based on lifetimes estimated from interband $\mathrm{B}(\mathrm{E} 2)$ 's computed from $\mathrm{B}\left(\mathrm{E} 2 ; 2_{\beta}^{+} \rightarrow 2_{g}^{+}\right)$and Clebsch-Gordan coefficients. We do not include $\rho^{2}(\mathrm{E} 0)$ values so-deduced, here. 
be natural candidates for $\beta$ vibrations, do not often exhibit significant $0_{g}^{+} \rightarrow 2_{\beta}^{+}$E2 strength and do not exhibit any obvious systematic behavior. The strongest $\mathrm{B}\left(\mathrm{E} 2 ; 0_{g}^{+} \rightarrow 2_{\beta}^{+}\right)$values that we could find in Nuclear Data Sheets are for ${ }^{158} \mathrm{Dy}\left(2.1^{5}\right),{ }^{170} \mathrm{Yb}\left(2.7^{6}\right),{ }^{174} \mathrm{Hf}\left(2.2^{6}\right)$, and ${ }^{232} \mathrm{Th}\left(2.0^{3}\right)$, where the values in single-particle units are given in parentheses. The weakest values that we could find (assuming that the lowest $\mathrm{K}^{\pi}=0^{+}$band is always the $\beta$-vibrational band) are typically $\sim 0.3$ s.p. units and as low as 0.06 s.p. units in ${ }^{178} \mathrm{Hf}$. However, it has recently been found ${ }^{90}$ that in ${ }^{166} \mathrm{Er}$ the third excited $0^{+}$state has $\mathrm{B}\left(\mathrm{E} 2 ; 0_{4}^{+} \rightarrow 2_{g}^{+}\right)=8.8^{9}$ s.p. units, suggesting that one should look higher in energy for $\beta$ vibrations. All of this raises the question : "What is the nature of the lowest $\mathrm{K}^{\pi}=0^{+}$band in deformed nuclei ?" This is briefly considered below.

The structure of excited $\mathrm{K}^{\pi}=0^{+}$bands in deformed nuclei has been discussed from a number of viewpoints : this is well summarized in two recent publications ${ }^{90,91}$ that provide, in the nucleus ${ }^{166} \mathrm{Er}$, the most complete view of positive-parity collective states for any deformed nucleus. It is emphasized in these studies of ${ }^{166} \mathrm{Er}$ that pair-type excitations must be important at low energy. Indeed, pair excitations must exist in a pair-correlated system, although the discussion of such states has received only limited attention theoretically (see, e.g. ${ }^{92}$ ).

Although a discussion of the above issues is beyond the scope of the present work, we note that a specific type of pair excitation can give rise to enhanced E0 strength. Namely, if a nucleon pair is excited from, e.g., a set of up-sloping Nilsson orbitals into a set of down-sloping Nilsson orbitals then configurations with different deformations are possible (evidence for such shape coexistence may be reflected by significant variations in rotational parameters for $K=0$ bands in deformed nuclei). If these configurations mix then E0 transition strength between the states resulting from the mixing will be observed. For complete mixing and $\beta$ values of 0.25 and 0.33 , one obtains from Equation (47), for $\mathrm{Z}=70$, the result $\rho^{2}(\mathrm{E} 0) \cdot 10^{3}=150$, and for $\mathrm{Z}=90$ the result $\rho^{2}(\mathrm{E} 0) \cdot 10^{3}=250$.

Within the descriptive framework of the interacting boson model, the SU(3) limit of IBM-1 has been applied $^{27}$ to deformed nuclei. In particular, ${ }^{168} \mathrm{Er}$ has been considered ${ }^{93}$ to be one of the best manifestations of $\mathrm{SU}(3)$ boson behavior in a deformed nucleus. The $\rho^{2}(\mathrm{E} 0)$ data for ${ }^{168} \operatorname{Er}$ (cf. Table 3) would therefore suggest that little if any (the value for ${ }^{168} \mathrm{Er}$ is consistent with zero) monopole transition strength should be associated with $\mathrm{SU}(3)$ boson states in the IBM. This point needs careful investigation (however, it is beyond the scope of the present work).

Finally, we comment on three very large values of $\rho^{2} \cdot 10^{3}$ in Table 3 , namely ${ }^{162} \operatorname{Er}\left(630^{460}\right),{ }^{238} \mathrm{U}$ $\left(176_{-32}^{+36}\right)$, and ${ }^{238} \mathrm{Pu}\left(180^{110}\right)$. The value for ${ }^{162} \mathrm{Er}$ depends upon a lifetime deduced from inelastic scattering of $\alpha$ particles ${ }^{94}$. The $\gamma$-branching ratios from the $1171 \mathrm{keV}$ state in question in ${ }^{162} \mathrm{Er}$, according to Nuclear Data Sheets, indicate a very weak E2 matrix element with the ground state and consequently very strong E2 matrix elements with the $2_{g}^{+}$and $4_{g}^{+}$states. A coupled-channels analysis of the inelastic scattering data would be desirable to determine if two-step excitation (via the $2_{g}^{+}$state) plays an important role. The value for ${ }^{238} \mathrm{U}$ depends upon a "mismatch" between $\left(\alpha, \alpha^{\prime}\right)$ and $\left(\alpha, \alpha^{\prime} \gamma\right)$ data ${ }^{95}$ which is reconciled by invoking unobserved internal conversion. A direct observation of this is desirable. The value for ${ }^{238} \mathrm{Pu}$ depends upon unresolved doublets in the $\gamma$-ray and conversion-electron spectra of ${ }^{238} \mathrm{~Np} \rightarrow{ }^{238} \mathrm{Pu}^{96}$ and of ${ }^{238} \mathrm{Am} \rightarrow{ }^{238} \mathrm{Pu}^{97}$ and it would be desirable to narrow the errors on the $\gamma$-ray and conversion-electron intensities.

\section{Nuclei That Exhibit a Sudden Change in Ground-State Structure}

There are two known regions where a sudden change in ground-state structure occurs in nuclei : these are between $\mathrm{N}=58$ and 60 and $\mathrm{N}=88$ and 90 . Nuclei for which there are $\rho^{2}(\mathrm{E} 0)$ values are shown in Figures 16-19 for $\mathrm{N}=58,60,88,90$, respectively. There exists clear evidence for large E0 strength associated with these nuclei. In particular, the value $\rho_{32}^{2}(\mathrm{E} 0) \cdot 10^{3}=185 \pm 50$ units in ${ }^{96} \mathrm{Sr}$ is one of the strongest known in any nucleus with $\mathrm{A} \geq 56$; and the values of $\rho_{21}^{2}(\mathrm{E} 0)$ in ${ }^{100} \mathrm{Zr}$ and ${ }^{102} \mathrm{Mo}$ also rank among the strongest known in heavy nuclei. It is natural to associate the origin of these large $\rho^{2}(\mathrm{E} 0)$ values with shape mixing where we have in mind a "crossing over" of weakly-deformed and strongly-deformed states as shown in Figures 6 and 7 .

In the $\mathrm{N}=58,60 \mathrm{Sr}, \mathrm{Zr}$, Mo nuclei, Equation (48) can be used in its more general from, viz.

$$
\rho_{f i}^{2}(E 0)=\left(\frac{3}{4 \pi}\right)^{2} Z^{2} a^{2}\left(1-a^{2}\right) \beta_{d e f}^{4},
$$

considering the mixing of a deformed $(\beta \simeq 0.35)$ and a spherical configuration with $a$ the mixing amplitude between the spherical and deformed configuration ${ }^{98}$. We show, in Figure 20, the results for the 2-level model calculation of $\rho^{2}(\mathrm{E} 0)$. On the lower axis (upper axis) the unperturbed (perturbed) energy difference of the two $0^{+}$levels is indicated. Values of $\rho^{2}(\mathrm{E} 0) \cdot 10^{3}$ up to 350 can result ${ }^{99}$. In Figure 21 we compare 
all data for the strongest $\rho^{2}(\mathrm{E} 0)$ values in the $\mathrm{Sr}, \mathrm{Zr}, \mathrm{Mo}, \mathrm{Ru}$ and $\mathrm{Pd}$ nuclei with neutron number $50 \leq \mathrm{N} \leq 64$. One clearly observes the largest $\rho^{2}(\mathrm{E} 0)$ values near $\mathrm{N}=58,60$ where experimental values up to $\rho^{2}(\mathrm{E} 0) \cdot 10^{3} \simeq 185$ are obtained. These results present compelling evidence for the presence and mixing of largely different nuclear shapes. The energy spectra in this $Z=40, N=54-62$ mass region is consistent with the coexistence (and mixing) of almost spherical and strongly prolate deformed shapes ${ }^{5,6,99,100}$.

The $\mathrm{N}=90$ nuclei are examples of good rotors and thus are well deformed. So, the question arises (cf. Section 2 C 2) : "Are the $0_{2}^{+}$states in the $\mathrm{N}=90$ nuclei $\beta$ vibrations?" We note that the spacing of the rotational bands built on the ground state and first excited $0^{+}$state in ${ }^{152} \mathrm{Sm},{ }^{154} \mathrm{Gd},{ }^{156}$ Dy are very similar, which would be characteristic of $\beta$ vibrations. However, we also note a peculiarity of the $\mathrm{B}(\mathrm{E} 2)$ values in the ground-state bands of the $\mathrm{N}=90$ nuclei, as compared to other well-deformed nuclei. This is shown in Figure 22. Usually, B(E2) values for ground-state bands of well-deformed nuclei are, within experimental error, in agreement with the rigid rotor model (unlike excitation energies!) However, unique to the $\mathrm{N}=90$ nuclei among well-deformed nuclei, the B(E2)'s of their ground-state bands clearly increase (more in accord with one's expectations cf. the deviation of excitation energies from a rigid rotor, i.e., in support of centrifugal stretching). To our knowledge, this feature of the $\mathrm{N}=90$ nuclei (and indeed of the conformity of ground-state band B(E2)'s to the rigid rotor for well-deformed nuclei cf. energies) has not been recognized. Thus, we incline towards an interpretation of the $\mathrm{N}=90$ nuclei as very soft, i.e., as involving mixing of configurations with different shapes. This needs further exploration. We note a set of calculations for ${ }^{154,156,158} \mathrm{Gd}^{101}$, that can be described as IBM-1 configuration mixing calculations, which discuss $\rho^{2}(\mathrm{E} 0)$ values in these nuclei. We further note a very recent interpretation ${ }^{102}$ of ${ }^{152} \mathrm{Sm}$ in terms of phase coexistence.

\section{E. Nuclei That Exhibit Shape Coexistence and Intruder States}

Intruder states are generally associated with shape coexistence. This topic has been explored thoroughly in two reviews ${ }^{5,103}$ to which we refer the reader for details of the evidence for shape coexistence.. Already in the review on doubly-even nuclei ${ }^{5}$, the qualitative connection between shape coexistence and E0 strength was made. This section and the previous section provide an updated and extended perspective of this connection.

In Figures 23 and 24 the $\rho^{2}$ (E0) data ${ }^{104-106}$ for the doubly-even Sn and Cd nuclei are given. One of the most distinct features is the very strong $\rho_{32}^{2}(\mathrm{E} 0)$ values, in particular in the Sn nuclei, which is a clear indication of strong mixing between an almost spherical and a deformed intruder excitation ${ }^{107}$. The $\mathrm{Cd}$ nuclei also exhibit strong $\rho_{32}^{2}(\mathrm{E} 0)$ values. For ${ }^{114} \mathrm{Cd}$, a detailed illustration of the relative E0 strength is given in Figure 25. The IBM-2 configuration mixing calculations of Heyde et al. ${ }^{108}$ are in fair agreement with observed values for $\rho^{2}(\mathrm{E} 0)$ in ${ }^{114} \mathrm{Cd}$.

The data for the $\mathrm{Z}=82$ mass region are shown for the $\mathrm{Pb}$ nuclei in Figure 26 and for the $\mathrm{Hg}$ nuclei in Figure $27^{5}$. Information on the mixing between the ground-state spherical and the intruder deformed configuration has been deduced for the $\mathrm{Pb}$ nuclei $(192 \leq \mathrm{A} \leq 196)^{109,110}$ and for the $\mathrm{Hg} \mathrm{nu}-$ clei $(184 \leq \mathrm{A} \leq 190)^{111,112}$. For the $\mathrm{Hg}$ nuclei $(180 \leq \mathrm{A} \leq 188)$, the mixing amplitude, deduced from $\rho^{2}(\mathrm{E} 0)$ values $^{111}$ and from $\alpha$-decay studies ${ }^{112}$, is given in Figure 28 . In this figure, a comparison with IBM-2 configuration mixing calculations ${ }^{113}$ is presented also. These calculations give a reasonable prediction of the $\rho^{2}(\mathrm{E} 0)$ values in ${ }^{186,188} \mathrm{Hg}$ from parameters fitted to isotope shift data.

\section{F. Light Nuclei $(\mathrm{A}<56)$}

We separate light nuclei from the detailed classifications of Sections 3 A-3 E because some $\rho^{2}($ E0) values appear significantly larger than those in heavier nuclei although this is simply an $\mathrm{A}^{-2 / 3}$ effect (see Equation 14).

In Table 4 we present the excitation energy, the $\rho^{2}(\mathrm{E} 0)$ values, as well as the 'reduced' $\rho^{2}(\mathrm{E} 0)$ values, i.e., $\rho^{2}(\mathrm{E} 0) \cdot A^{2 / 3}$ for selected light nuclei, where we also compare with some of the largest $\rho^{2}$ (E0) values.

From the shell-model analysis, as given in Section 2 B, within a given shell (e.g., the sd-shell), one does not expect strong $\rho^{2}(\mathrm{E} 0)$ values. It appears though that in a number of $\mathrm{N}=\mathrm{Z}$ sd-shell nuclei (see Figure $29)$, e.g., ${ }^{24} \mathrm{Mg}$ and ${ }^{28} \mathrm{Si}$, strong $\rho^{2}$ (E0) values are observed.

In light sd shell nuclei, in particular in ${ }^{16} \mathrm{O},{ }^{18} \mathrm{O}$ and ${ }^{20} \mathrm{Ne}$ clear-cut evidence for deformed $4 \mathrm{p}-4 \mathrm{~h}$ excitations across the $\mathrm{Z}, \mathrm{N}=8$ closed shell has been given (see, e.g., ${ }^{5}$ ). Further into the sd shell, near to $\mathrm{N}=\mathrm{Z}$, inelastic electron scattering on ${ }^{26} \mathrm{Mg}^{116}$ and inelastic $\alpha$-particle scattering on ${ }^{24} \mathrm{Mg}^{116}$ and ${ }^{28} \mathrm{Si}^{117}$ result in form factors which indicate the need for incorporating excitations out of the p-shell core and for $2 \hbar \omega$ excitations out of the sd shell, respectively. In such excitations, the radial extension of orbitals in the $\mathrm{N}=1$ and the $\mathrm{N}=3$ and 4 shells are quite different from those in the $\mathrm{N}=2$ shell, and this may partly explain 
the presence of these strong $\rho^{2}(\mathrm{E} 0)$ values. A more precise description will need a large-scale shell-model calculation where the sd-model space is expanded to include multi-particle multi-hole excitations in a consistent way. Attempts in this direction, albeit in a approximate way, have been carried out near the $\mathrm{N}=20$ shell-closure for sd-shell nuclei ${ }^{118-122}$.

Although we suggest in the previous paragraph that understanding strong $\rho^{2}(\mathrm{E} 0)$ values in sd-shell nuclei requires investigation, nuclei with $\mathrm{A}<56$ which exhibit ${ }^{5}$ shape coexistence possess moderate to strong $\rho^{2}(\mathrm{E} 0)$ values. The nuclei ${ }^{16,18} \mathrm{O},{ }^{38} \mathrm{Ar}$ and ${ }^{40,42,44} \mathrm{Ca}$ (cf. Figures 3.1-3.6, $3.9 \mathrm{in}^{5}$ ) are included in Table 4 .

\section{G. Odd-A Nuclei}

Recently, it has become evident that E0 transitions may exhibit widespread occurrence in odd-A nuclei (see, e.g., ${ }^{123^{-125}}$ ). The identification of E0 transitions in odd-mass nuclei is not new (see, e.g., ${ }^{126}$ ) ; but it is not easy because coincidence gating of conversion electrons is needed to correctly locate the E0 transitions in the scheme.

At present, we know of no $\rho^{2}$ (E0) data for odd-A nuclei. To obtain such data will be challenging because of the higher level density and consequent greater decay scheme complexity in odd-A nuclei. However, information in odd-mass nuclei would be of great value for understanding mixing mechanisms because of the restrictions on mixing due to the spin-parity of the unpaired nucleon (see for example a recent study of $\left.{ }^{187} \mathrm{Au}^{125}\right)$. Such information could cast light on the unusual E0 decay pattern in ${ }^{184,186} \mathrm{Pt}^{127,128}$ which are core nuclei for ${ }^{185} \mathrm{Pt}$ and ${ }^{187} \mathrm{Au}$.

\section{H. Relating $\rho^{2}$ and $\Delta\left\langle r^{2}\right\rangle$}

An important influence on this study has been the emerging broad picture of $\left\langle r^{2}\right\rangle$ information in nuclei provided by the atomic hyperfine spectroscopy studies of long chains of isotopes using lasers ${ }^{33}$. Because the nuclear radius directly presents information on the ground-state wave function, information on the nuclear ground-state density can be derived and thus knowledge of the particular shape and shape change can be determined in an almost model-independent way.

The $\mathrm{Pb}, \mathrm{Hg}$, $\mathrm{Pt}$ region provides a very clear illustration of this and is most dramatically illustrated in Figure 30 where the $\left\langle r^{2}\right\rangle$ values are presented for the $\mathrm{Hg}, \mathrm{Au}$ and $\mathrm{Pt}$ nuclei (both even- and odd-mass nuclei). Compared to the gentle variation of a spherical liquid-drop model, given by the expression of Equation 50, the jumps in the odd-mass $\mathrm{Hg}$ and the $\mathrm{Au}$ nuclei cannot so easily be understood. The explanation for the odd-even staggering effect in the $\mathrm{Hg}$ nuclei is related to a drastic change in the occupied single-particle orbitals for the ground state and isomeric state as situated in the small oblate and large prolate deformed minima of the energy surface ${ }^{5}$. The behaviour in the odd-mass Au nuclei $(\mathrm{Z}=79)$ is related to a lowering of orbitals from the $1 h_{9 / 2}$ configuration lying above the $\mathrm{Z}=82$ closed shell at deformation $\epsilon_{2}=0^{103}$. In a spherical model, the negative-parity configuration can be associated with a 4h-1p excited configuration as the intruding configuration that now looks more like a $\mathrm{Pt}$ core +1 particle. The change from a $\mathrm{Hg}$ core $+1 \mathrm{~h}$ into a $\mathrm{Pt}$ core $+1 \mathrm{p}$ can be analysed in terms of a change in $\beta^{2}$ value (in the region of $\mathrm{N}=108$ ) from $\beta_{2} \simeq 0.12$ in $\mathrm{Hg}$ nuclei to $\beta_{2}=0.21$ in Pt nuclei and so implies a large jump in the nuclear ground-state deformation and shape ${ }^{5,103}$.

The interest now is to go to even more neutron-deficient Au nuclei in this mass region. The likely extrapolation for the ground-state structure is as shown by the dotted lines in Figure 31 which makes the intruder Pt core $+1 \mathrm{p}(4 \mathrm{~h}-1 \mathrm{p})$ structure the ground-state $(98,100 \leq \mathrm{N} \leq 108)$ before changing back to the regular states originating from the $2 s_{1 / 2}, 1 d_{3 / 2}$ and $1 h_{11 / 2}$ orbitals. Experiments on $\left\langle r^{2}\right\rangle$ values in this very-neutron-deficient region would be most interesting to test the above ideas of shape changes through a modification of the core structure (from $\mathrm{Hg}$ to $\mathrm{Pt}$ and back to $\mathrm{Hg}$ ).

\section{Conclusions}

From the extensive discussion on E0 monopole properties carried out in the present paper, in which the whole nuclear mass region is considered, it becomes clear that the strongest E0 transitions $\left(\rho^{2}(\mathrm{E} 0) \cdot 10^{3} \geq\right.$ $20)$, at least for nuclei which do not have deformed ground states, are associated with the coexistence of configurations with different shapes and their mixing.

The strongest E0 transitions quite often result between pairs of excited states, i.e., the $0^{+}$ground state is not involved in the process.

When studying the variations in $\rho^{2}(\mathrm{E} 0)$ values within a given model type (single-closed shell nuclei, 
vibrational or weakly deformed systems, strongly deformed nuclei, ...), it is not easy to connect model variations to the corresponding experimental $\rho_{\text {exp }}^{2}(\mathrm{E} 0)$ values unless shape mixing effects have been removed. This immediately follows from the fact that shape admixtures totally dominate the E0 decay pattern.

One can definitely put the E0 $\left(\rho^{2}(\mathrm{E} 0)\right)$ observable forward as a very sensitive probe of deformed shape admixtures, i.e., it acts as a spectroscopic fingerprint for the presence of shape mixing in a region of nuclei.

We also point out that there is a serious lack of $T_{1 / 2}\left(0^{+}\right)$experimental values, at present. This is related to the many experimental difficulties when extending lifetime measurements into the region $T_{1 / 2}<10 \mathrm{ps}$. Here, a clear need for Doppler shift data emerges. A program of studies of the $\left(n, n^{\prime} \gamma\right)$ reaction $^{131,132}$ is very helpful, in this respect.

An intimate connection between E0 transitions (non-diagonal matrix elements) on the one hand, and isotopic shifts (differences of diagonal matrix elements) on the other hand has been pointed out, with ample illustration of its validity in the $\mathrm{Sr}, \mathrm{Zr}$, and in the $\mathrm{Pb}$ nuclei. This proves to be a good test to check consistency between observed E0 and $\left\langle r^{2}\right\rangle$ values in given mass regions.

At present, not enough data are available on $J^{\pi} \rightarrow J^{\pi}$ E0 transitions (with $J^{\pi} \neq 0^{+}$), to test a possible $J$ dependence in the resulting $\rho^{2}$ (E0) values. Such a result may shed light on the connection of the geometrical and algebraic collective models to nuclei in the rare-earth and actinide region.

Finally, relations between E0 properties (testing mean square change fluctuations in the atomic nucleus) and other multipole properties (M1,E2) have recently been suggested ${ }^{133,134}$. Further explorations and detailed studies along these lines are needed.

\section{Acknowledgments}

The authors would like to thank many people for suggesting that we try to present E0 monopole properties in a coherent way, discussing the underlying model concepts and making the full E0 data set available. We thank, in particular : H.J.Kluge and I.Talmi for discussions on the importance of relating $\rho^{2}(\mathrm{E} 0)$ values to isotopic shifts in the $\mathrm{Hg}$ region, in an early phase ; H.Mach for discussions regarding half-life measurements $\left(T_{1 / 2}\left(0^{+}\right)\right.$'s $)$; and J.Van Klinken for discussions about E0 measurements in the actinide region. The input from P.Van Isacker, M.Harder, W.Nazarewicz, T.Otsuka, J.Draayer, D.Troltenier, J.Escher, and P.H.Heenen on theoretical issues has been much appreciated.

This work was supported by DOE grant DE-FG02-96ER40958 at Georgia Tech. (JLW), DE-FG0296ER40978 at LSU(EFZ) and by a NATO Research Grant CRG 96-0981. Two of the authors (KH,CDC) thank the FWO-Flanders for financial support and one of them $(\mathrm{KH})$ is grateful to CERN for financial support during the latest stages of this work.

1. A.Bohr and B.Mottelson, Nuclear Structure (Benjamin, Reading, Massachusetts, 1975).

2. J.Eisenberg and W.Greiner, Nuclear Theory : Nuclear Models (North-Holland Publishing Co., Amsterdam, The Netherlands, 1987), Vol. 1.

3. J.Kantele, in Heavy Ions and Nuclear Structure, Proc. of the XIV Summer School, Mikolajki, 1984, edited by B.Sikora and Z.Wilhelmi (Harwood Academic Publ., New York, 1984), p. 391.

4. J.Kantele, Nucl. Instr. Meth. A271, 625 (1988).

5. J.L.Wood, K.Heyde, W.Nazarewicz, P. Van Duppen, and M.Huyse, Phys. Rep. 215, 101 (1992).

6. K.Heyde and R.A.Meyer, Phys. Rev. C37, 2170 (1988).

7. E.F.Zganjar and J.L.Wood, in Proc. Int. Conf. on Exotic Nuclei and Atomic Masses, ed. M. de Saint Simon and O.Sorlin (Editions Frontières, Gif-sur-Yvette, France, 1995), p. 437.

8. J.L.Wood, in Proc. Int. Conf. on Nuclear Shapes and Nuclear Structure at Low Excitation Energies, ed. M. Vergnes, D. Goutte, P.H.Heenen, and J. Sauvage (Editions Frontières, Gif-sur-Yvette, France, 1994), p. 295.

9. E.F.Zganjar and J.L.Wood, Nucl. Phys. A520, 427c (1990).

10. N.A.Voinova-Eliseeva and I. A. Mitropol'skii, Sov. J. Part. Nucl. 17, 521 (1986).

11. A.V.Aldushenkov and N.A.Voinova, Nucl. Data Tables 11, 299 (1972).

12. J.Schirmer, D.Habs, R.Kroth, N.Kwong, D.Schwalm, M.Zirnbauer, and C.Broude, Phys. Rev. Lett. 53, 1897 (1984). 
13. M.Nessin, T.H.Kruse, and K.E.Eklund, Phys. Rev. 125, 639 (1962).

14. D.A.Bell, C.E.Aveledo, M.G.Davidson, and J.P.Davidson, Can. J. Phys. 48, 2542 (1970).

15. E.L.Church and J.Weneser, Phys. Rev. 103, 1035 (1956).

16. G.G.Colvin and K.Schreckenbach, in Capture Gamma-Ray Spectroscopy and Related Topics, No. 125 in AIP Conf.Proc., ed. S.Raman (Am. Inst. Phys., N.Y., 1985), p. 290.

17. R.S.Hager and E.C.Seltzer, Nucl. Data A6, 1 (1969).

18. A.Passoja and T.Salonen, Report (Univ. of Jyväskylä) JYFL RR 2/86 .

19. D.H.Wilkinson, Nucl. Phys. A133, 1 (1969).

20. A.Moalem, Phys. Rev. C26, 1757 (1982).

21. D.Troltenier, J.Maruhn, W.Greiner, V. Aguilar, P.O.Hess, and J.H.Hamilton, Z.Phys. A338, 261 (1991).

22. D.Troltenier, J.Maruhn, W.Greiner, and P.O.Hess, Z.Phys. A343, 25 (1992).

23. J.O.Rasmussen, Nucl. Phys. 19, 85 (1960).

24. A.Arima and F.Iachello, Ann. Phys. 99, 253 (1976).

25. A.Arima and F.Iachello, Ann. Phys. 111, 201 (1978).

26. A.Arima and F.Iachello, Ann. Phys. 123, 468 (1979).

27. F.Iachello and A.Arima, The Interacting Boson Model (Cambridge University Press, Cambridge, U.K., 1987).

28. T.Otsuka, A.Arima, and F.Iachello, Nucl. Phys. A309, 1 (1978).

29. A.Frank and P.Van Isacker, Algebraic Methods in Molecular and Nuclear Structure Physics (J.Wiley and Sons, Inc., 1994).

30. B.R.Barrett and T.Otsuka, Phys. Rev. C46, 1735 (1992).

31. J.Kantele, W.Stöffl, L.E.Ussery, D.J.Decman, E.A.Henry, R.W.Hoff, L.G.Mann, and G.L.Struble, Phys. Rev. Lett. 51, 91 (1983).

32. J.Kantele, W.Stöff, L.E.Ussery, D.J.Decman, E.A.Henry, R.J.Estep, R.W.Hoff, and L.G.Mann, Phys. Rev. C29, 1693 (1984).

33. E.Otten, in Treatise on Heavy-Ion Science, ed. D.A.Bromley (Plenum Publ. Co, 1989), Vol. 8, p. 517.

34. J.L.Wood, E.F.Zganjar, and K.Heyde, Z.Phys. A353, 355 (1996).

35. F.Buchinger et al., Phys. Rev. C41, 2883 (1990); errata C42, 2754 (1990).

36. E.K.Warburton and D.E.Alburger, Phys. Lett. 36B, 38 (1971).

37. A.Passoja, R.Julin, J.Kantele, and M.Luontama, Nucl. Phys. A363, 399 (1981).

38. D.Evers, W.Assmann, K.Rudolph, S.J.Skorka, and P.Sperr, Nucl. Phys. A198, 268 (1972).

39. W.P.Alford, R.A.Lindgren, D.Elmore, and R.W.Boyd, ibid. A243, 269 (1975).

40. D.L.Hanson, N.Stein, J.W.Sunier, C.W.Woods, and O.Hansen, Nucl. Phys. A321, 471 (1979).

41. W.J.Courtney and H.T.Fortune, Phys. Lett. 41B, 4 (1972).

42. A.Warwick, R.Chapman, J.L.Durell, J.N.Mo, and S.Sen, Phys. Lett. 88B, 55 (1979).

43. R.Julin, J.Kantele, M.Luontama, and A.Passoja, Z. Phys. A303, 147 (1981).

44. H.Mach, E.K.Warburton, W.Krips, R.L.Gill, and M.Moszynski, Phys. Rev. C42, 568 (1990).

45. D.Burch, P.Russo, H.Swanson, and E.G.Adelberger, Phys. Lett. 40B, 357 (1972).

46. G.Lhersonneau, B.Pfeiffer, K.-L.Kratz, T.Engvist, P.P.Jauho, A.Jokinen, J.Kantele, M.Leino, J.M.Parmonen, H.Penttilä, J.Äystö, and the ISOLDE Collaboration, Phys. Rev. C49, 1379 (1994).

47. K.Kawade, G.Battistuzzi, H.Lawin, H.A.Selič, K.Sistemich, F.Schussler, E.Monnand, J.A.Pinston, B.Pfeiffer, and G.Jung, Z. Phys. A304, 293 (1982).

48. H.Mach, M.Moszynski, R.L.Gill, F.K.Wohn, J.A.Winger, J.C.Hill, G.Molnár, and K.Sistemich, Phys. Lett. 230B, 21 (1989).

49. A.M.Van den Berg, A.Saha, G.D.Jones, L.W.Put, and R.H.Siemssen, Nucl. Phys. A429, 1 (1984).

50. R.Julin, J.Kantele, M.Luontama, A.Passoja, P.Kleinheinz, and J.Blomqvist, Phys. Lett. 94B, 123 (1980).

51. R.Julin, M.Luontama, A.Passoja, and W.Trzaska, ATOMKI Kozlem. 26, 36 (1984).

52. P.F.Mantica Jr., B.E.Zimmerman, W.B.Walters, and K.Heyde, Phys. Rev. C43, 1696 (1991).

53. R.Julin, J.Kantele, M.Luontama, P.Poikolainen, and V.Rahkonen, Phys. Lett. 65B, 337 (1976).

54. J.W.Tape, E.G.Adelberger, D.Burch, and L.Zamick, Phys. Rev. Lett. 29, 878 (1972).

55. J.Kantele, M.Luontama, W.Trzaska, R.Julin, A.Passoja, and K.Heyde, Phys. Lett. 171B, 151 (1986).

56. J.Speth, L.Zamick, and P.Ring, Nucl. Phys. A232, 1 (1974).

57. C.W.Ma and W.W.True, Phys. Rev. C8, 2313 (1973).

58. L.E.Svensson, C.Fahlander, L.Hasselgren, A.Bäcklin, L.Westerberg, D.Cline, T.Czosnyka, C.Y.Wu, R.M.Diamond, and H.Kluge, Nucl. Phys. A584, 547 (1995).

59. L.E.Svensson, Ph.D. thesis, Univ. of Uppsala, 1989.

60. R.J.Estep, R.K.Sheline, D.J.Decman, E.A.Henry, L.G.Mann, R.A.Meyer, W.Stoeff, L.E.Ussery, and J.Kantele, Phys. Rev. C35, 1485 (1987).

61. M.Luontama, R.Julin, J.Kantele, A.Passoja, W.Trzaska, A.Bäcklin, N.-G.Jonsson, and L.Westerberg, Z. Phys. A324, 317 (1986).

62. S.Ohya, K.Katsumata, K.Miyazawa, N.Mutsuro, and A.Furusawa, J. Phys. Soc. Japan 48, 1070 (1980).

63. G.Colvin, F.Hoyler, and S.J.Robinson, J.Phys. G13, 191 (1981).

64. J.Kern, P.E.Garrett, J.Jolie, and H.Lehmann, Nucl. Phys. A593, 21 (1995).

65. M.Koike, H.Kawakami, K.Komura, and H.Yamada, Phys. Rev. C10, 1996 (1974). 
66. A.Giannatiempo, A.Passeri, A.Perego, and P.Sona, Phys. Rev. C33, 1024 (1986).

67. J.Stachel, P.Van Isacker, and K.Heyde, Phys. Rev. C25, 680 (1982).

68. D.Bucurescu, G.Cata, D.Cutoiu, G.Constantinescu, M.Ivascu, and N.V.Zamfir, Z. Phys. A324, 387 (1986).

69. K.Kim, A.Gelberg, T.Mizusaki, T.Otsuka, and P. von Brentano, Nucl. Phys. A604, 163 (1996).

70. J.Stachel, N.Kaffrell, E.Grosse, H.Emling, H.Folger, R.Kulessa, and D.Schwalm, Nucl. Phys. A383, 429 (1982).

71. A.Passoja, R.Julin, J.Kantele, J.Kumpulainen, M.Luontama, and W.Trzaska, Nucl. Phys. A438, 413 (1985).

72. J.Reisberg, H.Genz, J.Lange, A.Richter, and H.-W.Müller, Nucl. Phys. A280, 13 (1977).

73. A.Passoja, M.Vergnes, J.Kantele, and M.Luontama, Phys. Scr. T5, 147 (1983).

74. A.Passoja, R.Julin, J.Kantele, M.Luontama, and M.Vergnes, Nucl. Phys. A441, 261 (1985).

75. A.V.Ramayya, R.M.Ronningen, J.H.Hamilton, W.T.Pinkston, G.Garcia-Bermudez, R.L.Robinson, H.J.Kim, H.K.Carter, and W.E.Collins, Phys. Rev. C12, 1360 (1975).

76. A.Makishima, M.Ishii, M.Ohshima, M.Adachi, and H.Taketani, Nucl. Phys. A425, 1 (1984).

77. A.Giannatiempo, A.Nannini, A.Passeri, A.Perego, and P.Sona, Z. Phys. A325, 157 (1986).

78. C.Chandler et al., Phys. Rev. C56, R2924 (1997).

79. A.Giannatiempo, A.Nannini, A.Perego, P.Sona, M.J.G.Borge, O.Tengblad, and the ISOLDE Collatoration, Phys. Rev. C52, 2444 (1995).

80. A.Giannatiempo, A.Nannini, A.Perego, P.Sona, M.J.G.Borge, K.Riisager, O.Tengblad, and the ISOLDE Collaboration, Phys. Rev. C47, 521 (1993).

81. A.Zemel, T.Hageman, J.J.Hamill, and J. Klinken, Phys. Rev. C31, 1483 (1985).

82. P.Sarriguren, E.Moyade Guerra, A.Escuderos, and A.C.Cerrizo, Nucl. Phys. A635, 55 (1998).

83. R.F.Casten and J.A.Cizewski, Nucl. Phys. A309, 477 (1978).

84. M.P.Fewell, G.J.Gyapong, R.H.Spear, M.T.Esat, A.M.Baxter, and S.M.Burnett, Phys. Lett. 1578, 353 (1985).

85. M.K.Harder and B.Krusche, Phys. Lett. B331, 25 (1994).

86. P.F.Mantica and W.B.Walters, Phys. Rev. C53, R2586 (1996).

87. R.Bijker, A.E.L.Dieperink, O.Scholten, and R.Spanhoff, Nucl. Phys. A344, 207 (1980).

88. W.R.Kane, R.F.Casten, D.D.Warner, K.Schreckenbach, H.R.Faust, and S.Blakeway, Phys. Lett. 117B, 15 (1982).

89. H.Ejiri and G.B.Hageman, Nucl. Phys. A161, 449 (1971).

90. P.E.Garrett, M.Kadi, C.A.McGrath, V.Sorokin, M. Li, M.F.Yeh, and S.W.Yates, Phys. Lett. B400, 250 (1997).

91. P.E.Garrett, M.Kadi, M.Li, C.A.McGrath, V.Sorokin, M.F.Yeh, and S.W.Yates, Phys. Rev. Lett. 78, 4545 (1997).

92. A.A.Shihab-Eldin, J.O.Rasmussen, M.A.Stoyer, D.G.Burke, and P.E.Garrett, Int. J. Mod. Phys. E4, 411 (1995).

93. D.D.Warner, R.F.Casten, and W.F.Davidson, Phys. Rev. C24, 1713 (1981).

94. R.M.Ronningen et al., Phys. Rev. C26, 97 (1982).

95. F.K.McGowan and W.T.Milner, Nucl. Phys. A571, 569 (1994).

96. C.M.Lederer, Phys. Rev. C24, 1175 (1981).

97. I.Ahmad, R.K.Sjoblom, R.F.Barnes, F.Wagner Jr., and P.R.Fields, Nucl. Phys. A186, 620 (1972).

98. K.Heyde, E.D.Kirchuk, and P.Federman, Phys. Rev. C38, 984 (1988).

99. K.Heyde and R.A.Meyer, Phys. Rev. C42, 790 (1980).

100. H.Mach, M.Moszynski, R.L.Gill, G.Molnar, F.K.Wohn, J.A.Winger, and J.C.Hill, Phys. Rev. C42, 793 (1990).

101. P.Van Isacker, K.Heyde, M.Waroquier, and G.Wenes, Nucl. Phys. A380, 383 (1982).

102. F.Iachello, N.V.Zamfir, and R.F.Casten, Phys. Rev. Lett. 81, 1191 (1998).

103. K.Heyde, P.Van Isacker, M.Waroquier, J.L.Wood, and R.A.Meyer, Phys. Repts. 102, 291 (1983).

104. J.Kantele, R.Julin, M.Luontama, A.Passoja, T.Poikolainen, A.Bäcklin, and N.-G.Jonson, Z. Phys. A289, 157 (1979).

105. R.Julin, J.Kantele, M.Luontama, A.Passoja, T.Poikolainen, A.Bäcklin, and N.-G.Jonsson, Z. Phys. A296, 315 (1980).

106. A.Bäcklin, N.G.Jonson, R.Julin, J.Kantele, M.Luontama, A.Passoja, and T.Poikolainen, Nucl. Phys. A351, 490 (1981).

107. G.Wenes, P.Van Isacker, M.Waroquier, K.Heyde, and J.Van Maldeghem, Phys. Rev. C23, 2291 (1981).

108. K.Heyde, P.Van Isacker, M.Waroquier, G.Wenes, and M.Sambataro, Phys. Rev. C25, 3160 (1982).

109. P.Van Duppen, E.Coenen, K.Deneffe, M.Huyse, and J.L.Wood, Phys. Rev. C35, 1861 (1987).

110. P.Dendooven, P.Decrock, M.Huyse, G.Reusen, P.Van Duppen, and J.Wauters, Phys. Lett. B226, 27 (1989).

111. P.Joshi et al., Int. J. Mod. Phys. E3, 757 (1994).

112. J.Wauters, N.Bijnens, H.Folger, M.Huyse, H.Y.Hwang, R.Kirchner, J.Schwarzenberg, and P.Van Duppen, Phys. Rev. C50, 2768 (1994).

113. A.Barfield, B.R.Barrett, K.A.Sage, and P.D.Duval, Z. Phys. A311, 205 (1983).

114. P.M.Endt, At.Data Nucl. Data Tables 55, 171 (1993). 
115. P.M.Endt, Nucl. Phys. A521, 1 (1990).

116. H.Blok, H.P.Blok, J.F.A.van Hienen, G.van der Steenhoven, C.W.de Jager, H.de Vries, A.Saha, and K.K.Seth, Phys. Lett. 149B, 441 (1984).

117. H.P.Morsch, D.Dehnhard, and T.K.Li, Phys. Rev. Lett. 34, 1527 (1975).

118. W.C.Haxton and C.Johnson, Phys. Rev. Lett. 65, 1325 (1990).

119. E.K.Warburton, J.A.Becker, and B.A.Brown, Phys. Rev. C41, 1147 (1990).

120. K.Heyde and J.L.Wood, J. Phys. G17, 135 (1991).

121. N.Fukunishi, T.Otsuka, and T.Sebe, Phys. Lett. 296B, 279 (1992).

122. A.Poves and J.Retamosa, Nucl. Phys. A571, 221 (1994).

123. C.D.Papanicolopulos, M.A.Grimm, J.L.Wood, E.F.Zganjar, M.O.Kortelahti, J.D.Cole, and H.K.Carter, Z. Phys. A330, 371 (1988).

124. J.Schwarzenberg, J.L.Wood, and E.F.Zganjar, Phys. Rev. C45, R896 (1992).

125. D.Rupnik, E.F.Zganjar, J.L.Wood, P.B.Semmes, and W.Nazarewicz, Phys. Rev. C51, R2867 (1995).

126. E.F.Zganjar and J.L.Wood, in Proc. Int. Workshop on Nuclear Structure in the Zirconium Region, Bad Honef, Germany, April 24-28, 1988, ed. J.Eberth et al. (Springer Verlag, Berlin, 1988), p. 88.

127. Y.Xu, K.S.Krane, M.A.Gummin, M.Jarrio, J.L.Wood, E.F.Zganjar, and H.K.Carter, Rev. Lett. 68, 3853 (1992).

128. J.G.McEver, Ph.D. thesis, Georgia Tech., 1997.

129. Th.Hilberath, St.Becker, G.Bollen, H.-J.Kluge, U.Krönert, G.Passler, J.Rikovska, R.Wyss, and the ISOLDE Collaboration, Z.Phys. 342, 1 (1992).

130. G.Passler, J.Rikovska, E.Arnold, H.-J.Kluge, L.Monz, R.Neugart, H.Ravn, K.Wendt, and the ISOLDE Collaboration, Nucl. Phys. A580, 173 (1994).

131. S.W.Yates, A.Khan, A.J.Filo, M.C.Mirzaa, J.L.Weil, and M.T.McEllistrem, Nucl. Phys. A406, 519 (1983).

132. S.W.Yates, C.A.McGrath, P.E.Garrett, M.Yeh, and T.Belgya, in Proc. of the 9th Int. Conf. of Capture Gamma-Ray Spectroscopy and Related Topics, edited by G.L.Molnár, T.Belgya, and Z. Révay (Springer Verlag, Berlin, 1997), Vol. 1, p. 211 and refs therein.

133. P.von Neumann-Cosel, J.N.Ginocchio, H.Bauer, and A.Richter, Phys. Rev. Lett. 75, 4178 (1995).

134. A.Richter, Progr. Part. Nucl. Phys. 34, 261 (1995).

135. A.Passoja, J.Kantele, M.Luontama, R.Julin, E.Hammarén, P.O.Lipas, and P.Toivonen, J. Phys. G12, 1047 (1986).

136. H.Kawakami, N.Yoshikawa, K.Komura, M.Koike, and H.Yamada, Phys. Rev. C25, 2013 (1981).

137. A.Giannatiempo, A.Nannini, A.Perego, and P.Sona, Phys. Rev. C44, 1844 (1991).

138. B.Bengtson, H.L.Nielsen, N.Rud, and K.Wilsky, Nucl. Phys. A378, 1 (1982). 
Fig. 1. The spin dependence $(L)$ for E0 transitions connecting the various $J^{\pi}$ levels in the $\beta$-band and ground-band (axial rotor) compared to the corresponding IBM-1 SU(3) E0 transitions (cf. Equation (37)).

Fig. 2. Schematic potential energy surface as a function of the quadrupole deformation. We illustrate the 2-level model with an almost spherical and a strongly deformed shape $\left(0_{1}^{+}, 0_{2}^{+}\right)$. Schematic collective wave functions are drawn with dashed lines.

Fig. 3. The fission isomer E0 decay in ${ }^{238} \mathrm{U}$ that represents the slowest E0 transition $\mathrm{known}^{31,32}$. The figure is taken from ${ }^{5}$, with corrections from Nuclear Data Sheets. The excitation energies of the super-deformed band members are estimates based on the rotational parameter shown which is typical for this structure (see, e.g., ${ }^{5}$ ).

Fig. 4. Variation of $\rho^{2}(\mathrm{E} 0) \cdot 10^{3}$, Equation(48), as a function of the deformation variable $\beta$. Values are typical for the $\mathrm{A}=100-120$ mass region.

Fig. 5. The isotopic shift $\Delta\left\langle r^{2}\right\rangle$ for the Sr nuclei. The data are taken from ${ }^{35}$.

Fig. 6. Schematic view of the isotope shift $\Delta\left\langle r^{2}\right\rangle$ (middle part) and the $\rho$ (E0) values (lower part) for a two-level model (upper part) in which a spherical and a strongly-deformed configuration are present. In the left hand part, no mixing is implied when the two levels cross; in the right-hand part large mixing at the crossing point is implied.

Fig. 7. The upper part shows the crossing of two configurations (unperturbed and perturbed cases are drawn) with no mixing $(\mathrm{V}=0)$ and mixing $(\mathrm{V}=100 \mathrm{keV})$. In the lower part, the relative energies for various coupling strengths $(\mathrm{V}=0,100$ and $200 \mathrm{keV}$ ) are given. (The figure corresponds to a situation in the platinum isotopes.)

Fig. 8. Electric monopole transition strengths, $\rho^{2}(\mathrm{E} 0) \cdot 10^{3}$, observed ${ }^{36,37}$ in the nickel isotopes. The proton-pair excitations, $\pi 2 \mathrm{p}-2 \mathrm{~h}$, have been identified by ${ }^{56,58} \mathrm{Fe}\left({ }^{3} \mathrm{He}, \mathrm{n}\right){ }^{38,39}$ and ${ }^{56,58,60} \mathrm{Fe}\left({ }^{16} \mathrm{O},{ }^{12} \mathrm{C}\right){ }^{40}$ transfer reactions. Other level data are taken from the relevant Nuclear Data Sheets. (In this figure and hereafter, errors are indicated for $\rho^{2}$ values if either the error is large or $\rho^{2}$ is large. Errors for all $\rho^{2}$ values are given in Tables 1-4.)

Fig. 9. Electric monopole transition strengths, $\rho^{2}(\mathrm{E} 0) \cdot 10^{3}$, observed $^{43-48}$ in the zirconium isotopes. (See also Figures 16, 17.) Other level data taken from the relevant Nuclear Data Sheets.

Fig. 10. The $\rho_{21}$ (E0) values, corresponding to the $0_{2}^{+} \rightarrow 0_{1}^{+}$E0 transitions for ${ }^{90,92,94,96} \mathrm{Zr}$. The dot-dashed line indicates theoretical estimates based on Equations $(55,56)$ with empirically determined values of $\alpha$ and $\beta$ (see ${ }^{43,49}$ ) and a shell model estimate of $\Delta\left\langle r^{2}\right\rangle$; the cross marks an estimate for ${ }^{90} \mathrm{Zr}$ using an empirically determined value for $\Delta\left\langle r^{2}\right\rangle$.

Fig. 11. Electric monopole transition strengths, $\rho^{2}(\mathrm{E} 0) \cdot 10^{3}$, observed ${ }^{50,51}$ in the $\mathrm{N}=82$ isotones.

Fig. 12. The low-lying states in ${ }^{108,110} \mathrm{Pd}$ and associated $\mathrm{B}(\mathrm{E} 2)$ values. Thick bars are strong transitions. Forbidden transitions, in a harmonic quadrupole vibrator description, are marked by stars. The data are from ${ }^{58,59}$ and Nuclear Data Sheets.

Fig. 13. Electric monopole transition strengths, $\rho^{2}(\mathrm{E} 0) \cdot 10^{3}$, observed in the molybdenum ${ }^{60}$ (see also Figures $16,17)$, ruthenium ${ }^{65,66}$, and palladium ${ }^{60-63}$ isotopes. Other data are from ${ }^{58,59}$ and Nuclear Data Sheets.

Fig. 14. Electric monopole transition strengths, $\rho^{2}(\mathrm{E} 0) \cdot 10^{3}$, observed in the zinc $^{71,72}$, germanium ${ }^{73,74}$, selenium ${ }^{73,75^{-77}}$, and krypton ${ }^{78^{-81}}$, isotopes. Other data are from Nuclear Data Sheets. 
Fig. 15. Electric monopole transition strengths, $\rho^{2}(\mathrm{E} 0) \cdot 10^{3}$, for ${ }^{194,196} \mathrm{Pt}$. The data are taken from Harder and Krusche ${ }^{85}$ for ${ }^{196} \mathrm{Pt}$ and, for ${ }^{194} \mathrm{Pt}$, are derived using information given in the Nuclear Data Sheets for $\mathrm{A}=$ 194. Other $0^{+}$states and E0 transitions are shown for ${ }^{192-198} \mathrm{Pt}$. Other data are taken from Nuclear Data Sheets for $\mathrm{A}=192-198$. The dashed line boundaries for transitions in ${ }^{196} \mathrm{Pt}$ reflect different lower limits to half lives for the $0_{2}^{+}$and $0_{3}^{+}$states in ${ }^{196} \mathrm{Pt}$.

Fig. 16. Electric monopole transition strengths, $\rho^{2}(\mathrm{E} 0) \cdot 10^{3}$, observed in the $\mathrm{N}=58$ isotones. Also shown are $\mathrm{B}(\mathrm{E} 2)$ ratios which support the presence of coexisting collectivity in these nuclei. The data are from ${ }^{46,47}$ and Nuclear Data Sheets.

Fig. 17. Electric monopole transition strengths, $\rho^{2}(\mathrm{E} 0) \cdot 10^{3}$, observed in the $\mathrm{N}=60$ isotones. Also shown are $\mathrm{B}(\mathrm{E} 2)$ ratios which support the presence of weak coexisting collectivity in these nuclei. The data $\operatorname{are~}^{4} \mathrm{from}^{48,60}$ and Nuclear Data Sheets.

Fig. 18. Electric monopole transition strengths, $\rho^{2}(\mathrm{E} 0) \cdot 10^{3}$, observed in the $\mathrm{N}=88$ isotones. The data are from $^{135}$ and Nuclear Data Sheets.

Fig. 19. Electric monopole transition strengths, $\rho^{2}(\mathrm{E} 0) \cdot 10^{3}$, observed in the $\mathrm{N}=90$ isotones. The data are from Nuclear Data Sheets.

Fig. 20. The value of $\rho_{21}^{2}$ (E0), according to eq.(51) using a mixing matrix element $\left\langle H_{m i x}\right\rangle=100 \mathrm{keV}$. On the lower (upper) horizontal axis we denote the energy difference between unperturbed (perturbed) $0^{+}$states.

Fig. 21. Experimental results for $\rho^{2}(\mathrm{E} 0)$ in the $\mathrm{A} \simeq 100$ mass region. The data are taken from the present compilation and also are partially presented in ${ }^{6}$.

Fig. 22. Plot of the quantity $\mathrm{B}(\mathrm{E} 2)_{\text {yrast }} / \mathrm{B}(\mathrm{E} 2)_{\text {rotor }}$ (normalized at $\mathrm{I}=2$ ) for the averaged values of the $\mathrm{N}=90$ isotones $\left({ }^{150} \mathrm{Nd},{ }^{152} \mathrm{Sm},{ }^{154} \mathrm{Gd},{ }^{156} \mathrm{Dy},{ }^{158} \mathrm{Er}\right)$, open circles, compared to the averaged values of all (36) rare earth and actinide nuclei, filled circles and the $\mathrm{N}=92$ isotones, crosses. The data are taken from Nuclear Data Sheets. The line is drawn to guide the eye.

Fig. 23. Electric monopole transition strengths, $\rho^{2}(\mathrm{E} 0) \cdot 10^{3}$, observed in the Sn isotopes. Also shown are $\mathrm{B}(\mathrm{E} 2)$ ratios which support the identification ${ }^{5}$ of deformed bands in these nuclei. The data are from ${ }^{104,106,136}$ and Nuclear Data Sheets.

Fig. 24. Electric monopole transition strengths, $\rho^{2}(\mathrm{E} 0) \cdot 10^{3}$, observed in the Cd isotopes. Also shown are $\mathrm{B}(\mathrm{E} 2)$ ratios which support the identification ${ }^{5}$ of deformed bands in these nuclei. The data are from ${ }^{5,104,137}$ and Nuclear Data Sheets.

Fig. 25. Electric monopole transition strengths, $\rho^{2}(\mathrm{E} 0) \cdot 10^{3}$, observed in ${ }^{114} \mathrm{Cd}$. The data are from ${ }^{5}$ and Nuclear Data Sheets.

Fig. 26. Electric monopole transition strengths, $\rho^{2}(\mathrm{E} 0) \cdot 10^{3}$, observed in the light $\mathrm{Pb}$ isotopes. Also shown are $\mathrm{B}(\mathrm{E} 2)$ ratios which support the identification ${ }^{5}$ of deformed bands in these nuclei. The data are from ${ }^{110}$ and Nuclear Data Sheets.

Fig. 27. Electric monopole transition strengths, $\rho^{2}(\mathrm{E} 0) \cdot 10^{3}$, observed in the $\mathrm{Hg}$ isotopes. The data are from $^{111}$ and Nuclear Data Sheets. 
Fig. 28. Mixing amplitudes $\left(a^{2}\right)$ between the oblate and intruder prolate $0^{+}$band members in the even-even $\mathrm{Hg}$ nuclei, deduced from experimental $\rho^{2}$ values and $\alpha$-decay rates (filled circles). See text for references and theoretical values derived from IBM-2 configuration mixing calculations ${ }^{113}$ (filled squares).

Fig. 29. The low-lying states in ${ }^{24} \mathrm{Mg},{ }^{28} \mathrm{Si}$, and ${ }^{32} \mathrm{~S}$ organized into band structures. The basis of the band assignments is the $\mathrm{B}(\mathrm{E} 2)$ values which are shown in Weisskopf units in the boxes attatched to the relevant transitions. The known E0 transitions are shown as heavier lines and $\rho^{2}(\mathrm{E} 0) \cdot 10^{3}$ values are shown in attached boxes. Excitation energies are given in $\mathrm{keV}$. The data are taken from ${ }^{114,115}$

Fig. 30. The charge mean square radii $\left\langle r^{2}\right\rangle\left(\mathrm{fm}^{2}\right)$ for the even and odd-mass Pt, Au, and Hg nuclei (the data are taken from $^{33,129,130}$ ).

Fig. 31. Schematic variation of the mean-square charge radius for a spherical liquid drop (thin dashed line). The full line indicates the observed behavior for the odd-mass Au nuclei. The thick dashed line represents the expected behavior for the lighter odd-mass Au nuclei. 
Table 1. The strongest $\rho^{2}(\mathrm{E} 0)$ values in nuclei with weakly-deformed ${ }^{a}$ ground states

\begin{tabular}{|c|c|c|c|c|c|}
\hline Isotope & $\rho^{2}(E 0) \cdot 10^{3}$ & Trans. & Class $^{c}$ & Fig. & Reference \\
\hline${ }^{96} \mathrm{Sr}$ & $185(50)$ & $0_{3} \rightarrow 0_{2}$ & B & 16 & {$[\mathrm{NDS}]$} \\
\hline${ }^{152} \mathrm{Sm}$ & $\approx 150^{b}$ & $4_{2} \rightarrow 4_{1}$ & B & 19 & {$[\mathrm{NDS}]$} \\
\hline${ }^{102} \mathrm{Mo}$ & $120(50)$ & $0_{2} \rightarrow 0_{1}$ & B & 13,17 & \\
\hline${ }^{150} \mathrm{Sm}$ & $100(40)$ & $2_{2} \rightarrow 2_{1}$ & B & 18 & 135 \\
\hline${ }^{100} \mathrm{Zr}$ & $100(30)$ & $0_{2} \rightarrow 0_{1}$ & B & 9,17 & {$[\mathrm{NDS}]$} \\
\hline${ }^{154} \mathrm{Gd}$ & $96(17)^{b}$ & $0_{2} \rightarrow 0_{1}$ & $\mathrm{~B}$ & 19 & [NDS] \\
\hline${ }^{114} \mathrm{Cd}$ & $95(19)$ & $2_{4} \rightarrow 2_{2}$ & $\mathrm{~A}$ & 25 & \\
\hline${ }^{74} \mathrm{Kr}$ & $90(20)$ & $0_{2} \rightarrow 0_{1}$ & $\mathrm{C}$ & 14 & 78 \\
\hline${ }^{116} \mathrm{Sn}$ & $86(15)^{b}$ & $0_{3} \rightarrow 0_{2}$ & $\mathrm{~A}$ & 23 & {$[\mathrm{NDS}],{ }^{104},{ }^{106}$} \\
\hline${ }^{58} \mathrm{Ni}$ & $80(32)$ & $0_{3} \rightarrow 0_{1}$ & A & 8 & \\
\hline${ }^{62} \mathrm{Ni}$ & $78(43)$ & $0_{3} \rightarrow 0_{1}$ & $\mathrm{~A}$ & 8 & 37 \\
\hline${ }^{60} \mathrm{Ni}$ & $77(42)$ & $0_{2} \rightarrow 0_{1}$ & $\mathrm{~A}$ & 8 & 37 \\
\hline${ }^{98} \mathrm{Zr}$ & $75(8)$ & $\mathrm{O}_{3} \rightarrow \mathrm{O}_{2}$ & B & 9,16 & 46 \\
\hline${ }^{154} \mathrm{Gd}$ & $74(9)^{b}$ & $2_{2} \rightarrow 2_{1}$ & B & 19 & {$[\mathrm{NDS}]$} \\
\hline${ }^{152} \mathrm{Sm}$ & $69(6)^{b}$ & $2_{2} \rightarrow 2_{1}$ & B & 19 & {$[\mathrm{NDS}]$} \\
\hline${ }^{114} \mathrm{Cd}$ & $67(10)$ & $4_{2} \rightarrow 4_{1}$ & $\mathrm{~A}$ & 25 & \\
\hline${ }^{152} \mathrm{Gd}$ & $59(13)^{b}$ & $0_{2} \rightarrow 0_{1}$ & B & 18 & {$[\mathrm{NDS}]$} \\
\hline${ }^{152} \mathrm{Sm}$ & $58(6)^{b}$ & $0_{2} \rightarrow 0_{1}$ & B & 19 & {$[\mathrm{NDS}]$} \\
\hline${ }^{98} \mathrm{Zr}$ & $56(12)$ & $0_{4} \rightarrow 0_{2}$ & B & 9,16 & 46 \\
\hline${ }^{98} \mathrm{Sr}$ & $53(9)$ & $0_{2} \rightarrow 0_{1}$ & B & 17 & 48 \\
\hline${ }^{78} \mathrm{Kr}$ & $47(13)$ & $0_{2} \rightarrow 0_{1}$ & $\mathrm{C}$ & 14 & 79 \\
\hline${ }^{100} \mathrm{Mo}$ & $42(6)$ & $0_{2} \rightarrow 0_{1}$ & B & 13,16 & {$[\mathrm{NDS}]$} \\
\hline${ }^{76} \mathrm{Se}$ & $38(15)$ & $0_{2} \rightarrow 0_{1}$ & $\mathrm{C}$ & 14 & 73,77 \\
\hline${ }^{112} \mathrm{Cd}$ & $37(11)$ & $0_{2} \rightarrow 0_{1}$ & $\mathrm{~A}$ & 24 & {$[\mathrm{NDS}]$} \\
\hline${ }^{114} \mathrm{Cd}$ & $36(5)$ & $2_{2} \rightarrow 2_{1}$ & $\mathrm{~A}$ & 25 & \\
\hline${ }^{152} \mathrm{Gd}$ & $35(3)^{b}$ & $2_{2} \rightarrow 2_{1}$ & B & 18 & {$[\mathrm{NDS}]$} \\
\hline${ }^{74} \mathrm{Se}$ & $34(3)$ & $0_{2} \rightarrow 0_{1}$ & $\mathrm{C}$ & 14 & 73,76 \\
\hline${ }^{186} \mathrm{Hg}$ & $>32$ & $0_{2} \rightarrow 0_{1}$ & A & 27 & 111 \\
\hline${ }^{112} \mathrm{Cd}$ & $31(20)$ & $2_{3} \rightarrow 2_{2}$ & $\mathrm{~A}$ & 24 & 137 \\
\hline${ }^{72} \mathrm{Se}$ & $31(18)$ & $0_{2} \rightarrow 0_{1}$ & $\mathrm{C}$ & 14 & 73,74 \\
\hline${ }^{98} \mathrm{Mo}$ & $27(5)$ & $0_{2} \rightarrow 0_{1}$ & B & 13 & 10 \\
\hline${ }^{114} \mathrm{Sn}$ & $26(13)$ & $0_{2} \rightarrow 0_{1}$ & A & 23 & 106 \\
\hline${ }^{82} \mathrm{Kr}$ & $23\left({ }_{-17}^{+28}\right)^{b}$ & $0_{3} \rightarrow 0_{1}$ & $\mathrm{C}$ & 14 & {$[\mathrm{NDS}]{ }^{81}$} \\
\hline${ }^{152} \mathrm{Sm}$ & $22(9)^{b}$ & $0_{3} \rightarrow 0_{2}$ & B & 19 & {$[\mathrm{NDS}]$} \\
\hline${ }^{114} \mathrm{Cd}$ & $22(3)$ & $2_{3} \rightarrow 2_{2}$ & $\mathrm{~A}$ & 24,25 & 5 \\
\hline${ }^{80} \mathrm{Kr}$ & $21(9)$ & $0_{2} \rightarrow 0_{1}$ & $\mathrm{C}$ & 14 & 80 \\
\hline${ }^{110} \mathrm{Cd}$ & $20(15)$ & $2_{3} \rightarrow 2_{2}$ & $\mathrm{~A}$ & 24 & 137 \\
\hline
\end{tabular}

a.The $\mathrm{N}=60$ nuclei, ${ }^{152} \mathrm{Sm},{ }^{154} \mathrm{Gd}$ are also included (see text for further discussion).

b. Evaluation of data given in Nuclear Data Sheets and/or the cited references made by the authors.

c. Classification is made into : A) nuclei that exhibit shape coexistence and intruder states and lie at or near closed shells; B) nuclei that exhibit a sudden change in ground-state structure; and, C) nuclei that exhibit shape coexistence and lie far from closed shells. 


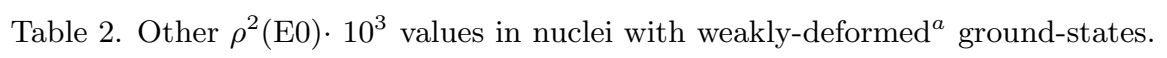

\begin{tabular}{|c|c|c|c|c|}
\hline Isotope & $\rho^{2}(\mathrm{E} 0) \cdot 10^{3}$ & Trans. & Fig. & Reference \\
\hline${ }^{58} \mathrm{Ni}$ & $0.0062(12)$ & $0_{2} \rightarrow 0_{1}$ & 8 & 36,37 \\
\hline${ }^{64} \mathrm{Zn}$ & $3.9(4)$ & $0_{2} \rightarrow 0_{1}$ & 14 & $71^{\prime}$ \\
\hline${ }^{64} \mathrm{Zn}$ & $8(3)$ & $0_{3} \rightarrow 0_{1}$ & 14 & 71 \\
\hline${ }^{68} \mathrm{Zn}$ & $7\left(\left(_{-4}^{+10}\right)\right.$ & $0_{2} \rightarrow 0_{1}$ & 14 & 71 \\
\hline${ }^{70} \mathrm{Zn}$ & $<2.6$ & $0_{2} \rightarrow 0_{1}$ & 14 & 71 \\
\hline${ }^{70} \mathrm{Ge}$ & $6.3(4)$ & $0_{2} \rightarrow 0_{1}$ & 14 & 73,74 \\
\hline${ }^{72} \mathrm{Ge}$ & $8.6(4)^{b}$ & $0_{2} \rightarrow 0_{1}$ & 14 & {$[\mathrm{NDS}]$} \\
\hline${ }^{82} \mathrm{Kr}$ & $9(3)^{b}$ & $0_{2} \rightarrow 0_{1}$ & 14 & {$[\mathrm{NDS}],{ }^{81}$} \\
\hline${ }^{90} \mathrm{Zr}$ & $3.30(17)$ & $0_{2} \rightarrow 0_{1}$ & 9 & 43,45 \\
\hline${ }^{92} \mathrm{Zr}$ & $8.1(8)^{b}$ & $0_{2} \rightarrow 0_{1}$ & 9 & 43,44 \\
\hline${ }^{94} \mathrm{Zr}$ & $11.5(11)^{b}$ & $0_{2} \rightarrow 0_{1}$ & 9 & 43,44 \\
\hline${ }^{96} \mathrm{Zr}$ & $7.4(3)^{b}$ & $0_{2} \rightarrow 0_{1}$ & 9 & 43,44 \\
\hline${ }^{98} \mathrm{Zr}$ & $10.4(12)$ & $0_{2} \rightarrow 0_{1}$ & 9,16 & {$[\mathrm{NDS}]$} \\
\hline${ }^{96} \mathrm{Mo}$ & $12(5)$ & $0_{2} \rightarrow 0_{1}$ & 13 & \\
\hline${ }^{100} \mathrm{Ru}$ & $11(2)$ & $0_{2} \rightarrow 0_{1}$ & 13 & {$[\mathrm{NDS}]$} \\
\hline${ }^{102} \mathrm{Ru}$ & $14(3)$ & $0_{2} \rightarrow 0_{1}$ & 13 & \\
\hline${ }^{102} \mathrm{Pd}$ & $4.0(15)$ & $0_{2} \rightarrow 0_{1}$ & 13 & 61 \\
\hline${ }^{102} \mathrm{Pd}$ & $<0.3$ & $0_{3} \rightarrow 0_{1}$ & 13 & 61 \\
\hline${ }^{104} \mathrm{Pd}$ & $4.7(20)$ & $0_{2} \rightarrow 0_{1}$ & 13 & 61 \\
\hline${ }^{106} \mathrm{Pd}$ & $14(3)^{b}$ & $0_{2} \rightarrow 0_{1}$ & 13 & 58,63 \\
\hline${ }^{108} \mathrm{Pd}$ & $<3^{b}$ & $0_{2} \rightarrow 0_{1}$ & 13 & 58,60 \\
\hline${ }^{110} \mathrm{Pd}$ & $3.4(6)^{b}$ & $0_{2} \rightarrow 0_{1}$ & 13 & 59,60 \\
\hline${ }^{110} \mathrm{Pd}$ & $0.48(15)^{b}$ & $0_{3} \rightarrow 0_{1}$ & 13 & {$[\mathrm{NDS}],{ }^{59}, 60$} \\
\hline${ }^{112} \mathrm{Cd}$ & $0.48(11)$ & $0_{3} \rightarrow 0_{1}$ & 24 & [NDS] \\
\hline${ }^{112} \mathrm{Cd}$ & $8.1(19)$ & $0_{3} \rightarrow 0_{2}$ & 24 & [NDS] \\
\hline${ }^{114} \mathrm{Cd}$ & $16(1)$ & $0_{2} \rightarrow 0_{1}$ & 24,25 & \\
\hline${ }^{114} \mathrm{Cd}$ & $1.3(1)$ & $0_{3} \rightarrow 0_{1}$ & 24,25 & 5 \\
\hline${ }^{114} \mathrm{Cd}$ & $0.7(3)$ & $0_{4} \rightarrow 0_{1}$ & 25 & 5 \\
\hline${ }^{114} \mathrm{Cd}$ & $0.45(4)$ & $0_{3} \rightarrow 0_{2}$ & 24,25 & 5 \\
\hline${ }^{116} \mathrm{Sn}$ & $4.4(13)$ & $0_{2} \rightarrow 0_{1}$ & 23 & 106 \\
\hline${ }^{116} \mathrm{Sn}$ & $0.83(15)^{b}$ & $0_{3} \rightarrow 0_{1}$ & 23 & {$[\mathrm{NDS}],{ }^{106}$} \\
\hline${ }^{118} \mathrm{Sn}$ & $5.2(13)$ & $0_{2} \rightarrow 0_{1}$ & 23 & 17 \\
\hline${ }^{120} \mathrm{Sn}$ & $2.6(7)$ & $0_{2} \rightarrow 0_{1}$ & 23 & 17 \\
\hline${ }^{140} \mathrm{Ce}$ & $14(4)$ & $0_{2} \rightarrow 0_{1}$ & 11 & 51 \\
\hline${ }^{142} \mathrm{Nd}$ & $17(6)$ & $0_{2} \rightarrow 0_{1}$ & 11 & 51 \\
\hline${ }^{146} \mathrm{Gd}$ & $10(1)$ & $0_{2} \rightarrow 0_{1}$ & 11 & 50,51 \\
\hline${ }^{150} \mathrm{Sm}$ & $18(3)$ & $0_{2} \rightarrow 0_{1}$ & 18 & 135 \\
\hline${ }^{152} \mathrm{Sm}$ & $0.7(4)^{b}$ & $0_{3} \rightarrow 0_{1}$ & 19 & [NDS] \\
\hline${ }^{188} \mathrm{Os}$ & $0.013(5)^{b}$ & $0_{2} \rightarrow 0_{1}$ & & [NDS] \\
\hline${ }^{188} \mathrm{Os}$ & $0.7(6)^{b}$ & $2_{2} \rightarrow 2_{1}$ & & [NDS] \\
\hline${ }^{194} \mathrm{Pt}$ & $0.16(8)^{b}$ & $0_{2} \rightarrow 0_{1}$ & 15 & [NDS] \\
\hline${ }^{194} \mathrm{Pt}$ & $11(4)^{b}$ & $0_{4} \rightarrow 0_{1}$ & 15 & [NDS] \\
\hline${ }^{194} \mathrm{Pt}$ & $0.46(16)^{b}$ & $2_{2} \rightarrow 2_{1}$ & & [NDS] \\
\hline${ }^{196} \mathrm{Pt}$ & $<0.07$ & $0_{2} \rightarrow 0_{1}$ & 15 & {$[\mathrm{NDS}]{ }^{85}$} \\
\hline${ }^{196} \mathrm{Pt}$ & $1.0(6)^{b}$ & $2_{2} \rightarrow 2_{1}$ & & [NDS] \\
\hline${ }^{188} \mathrm{Hg}$ & $7.7(25)$ & $0_{2} \rightarrow 0_{1}$ & 27 & {$[\mathrm{NDS}],{ }^{111}$} \\
\hline${ }^{192} \mathrm{~Pb}$ & $1.7(2)$ & $0_{2} \rightarrow 0_{1}$ & 26 & 110 \\
\hline${ }^{194} \mathrm{~Pb}$ & $1.0(2)$ & $0_{2} \rightarrow 0_{1}$ & 26 & 110 \\
\hline${ }^{204} \mathrm{~Pb}$ & $0.074(25)$ & $0_{2} \rightarrow 0_{1}$ & & 55 \\
\hline${ }^{206} \mathrm{~Pb}$ & $1.16(8)$ & $0_{2} \rightarrow 0_{1}$ & & 53,54 \\
\hline${ }^{208} \mathrm{Po}$ & $1.1(3)$ & $0_{2} \rightarrow 0_{1}$ & & $53^{\prime}$ \\
\hline${ }^{212} \mathrm{Po}$ & $1.2(2)$ & $0_{2} \rightarrow 0_{1}$ & & 138 \\
\hline${ }^{214} \mathrm{Po}$ & $1.48(7)^{b}$ & $0_{2} \rightarrow 0_{1}$ & & [NDS] \\
\hline
\end{tabular}

a.The $\mathrm{N}=60$ nuclei, ${ }^{152} \mathrm{Sm}$ and ${ }^{154} \mathrm{Gd}$ are also included.

b. Evaluation of data given in Nuclear Data Sheets and/or the cited references made by the authors. 
Table 3. $\rho^{2}(\mathrm{E} 0) \cdot 10^{3}$ values $^{a}$ in nuclei with deformed ground states

\begin{tabular}{|c|c|c|c|c|}
\hline Isotope & $\rho^{2}(\mathrm{E} 0) \cdot 10^{3}$ & $\begin{array}{c}\text { Trans. } \\
\mathrm{E}_{i} \rightarrow E_{f}\end{array}$ & $\mathrm{~J}, \mathrm{~K}_{i}^{\pi}$ & $\begin{array}{c}\text { Reference } \\
\text { (see footnotes) }\end{array}$ \\
\hline${ }^{156} \mathrm{Gd}$ & $55(5)$ & $1129 \rightarrow 89$ & $2,0_{2}^{+}$ & \\
\hline${ }^{156} \mathrm{Gd}$ & $0.2\left({ }_{-2}^{+6}\right)$ & $1258 \rightarrow 89$ & $2,0_{3}^{+}$ & \\
\hline${ }^{158} \mathrm{Gd}$ & $\leq 0.8$ & $1260 \rightarrow 79$ & $2,0_{2}^{+}$ & $78 \mathrm{Gr}$ \\
\hline${ }^{158} \mathrm{Gd}$ & $\overline{17}(3)$ & $1517 \rightarrow 79$ & $2,0_{3}^{+}$ & $78 \mathrm{Gr}$ \\
\hline${ }^{158} \mathrm{Dy}$ & $27(12)$ & $1086 \rightarrow 99$ & $2,0_{2}^{+}$ & $82 \mathrm{Ro}, 68 \mathrm{Gr}$ \\
\hline${ }^{160} \mathrm{Dy}$ & $17(4)$ & $1350 \rightarrow 87$ & $2,0_{2}^{+}$ & \\
\hline${ }^{162} \mathrm{Er}$ & $630(460)$ & $1171 \rightarrow 102$ & $2,0_{2}^{+}$ & \\
\hline${ }^{162} \mathrm{Er}$ & $40(30)$ & $1430 \rightarrow 102$ & $2, ?^{+}$ & \\
\hline${ }^{164} \mathrm{Er}$ & $5.3(27)$ & $1314 \rightarrow 91$ & $2,0_{2}^{+}$ & $82 \mathrm{Ro}$ \\
\hline${ }^{164} \mathrm{Er}$ & $90(50)$ & $1484 \rightarrow 91$ & $2,0_{3}^{+}$ & $82 \mathrm{Ro}$ \\
\hline${ }^{166} \mathrm{Er}$ & $2.0(10)$ & $1460 \rightarrow 0$ & $0,0_{2}^{+}$ & $97 \mathrm{Ga}$ \\
\hline${ }^{168} \mathrm{Er}$ & $0.8(8)$ & $1276 \rightarrow 80$ & $2,0_{2}^{+}$ & 81Da,98Le \\
\hline${ }^{168} \mathrm{Yb}$ & $30(7)$ & $1233 \rightarrow 78$ & $2,0_{2}^{+}$ & 70Ch,82Ro,66Gr \\
\hline${ }^{170} \mathrm{Yb}$ & $\leq 0.9$ & $1146 \rightarrow 85$ & $2,0_{2}^{+}$ & $72 \mathrm{Ca}$ \\
\hline${ }^{170} \mathrm{Yb}$ & $27(5)$ & $1229 \rightarrow 0$ & $0,0_{3}^{+}$ & \\
\hline${ }^{172} \mathrm{Yb}$ & $2.8(8)$ & $1043 \rightarrow 0$ & $0,0_{2}^{+}$ & \\
\hline${ }^{172} \mathrm{Yb}$ & $3.1(15)$ & $1118 \rightarrow 79$ & $2,0_{2}^{+}$ & $85 \mathrm{Ge}, 88 \mathrm{Su}$ \\
\hline${ }^{172} \mathrm{Yb}$ & $0.20(3)$ & $1406 \rightarrow 0$ & $0,0_{3}^{+}$ & \\
\hline${ }^{172} \mathrm{Yb}$ & $1.1(2)$ & $1406 \rightarrow 1043$ & $0,0_{3}^{+}$ & \\
\hline${ }^{172} \mathrm{Yb}$ & $\leq 0.013$ & $1477 \rightarrow 79$ & $2,0_{3}^{+}$ & $85 \mathrm{Ge}$ \\
\hline${ }^{172} \mathrm{Yb}$ & $9(6)$ & $1849 \rightarrow 79$ & $2,0_{4}^{+}$ & $85 \mathrm{Ge}$ \\
\hline${ }^{172} \mathrm{Yb}$ & $3.3(17)$ & $1956 \rightarrow 78$ & $2,0_{5}^{+}$ & $85 \mathrm{Ge}$ \\
\hline${ }^{174} \mathrm{Hf}$ & $27(13)$ & $900 \rightarrow 91$ & $2,0_{2}^{+}$ & $71 \mathrm{Ch}, 75 \mathrm{Ca}$ \\
\hline${ }^{176} \mathrm{Hf}$ & $52(9)$ & $1227 \rightarrow 89$ & $2,0_{2}^{+}$ & \\
\hline${ }^{178} \mathrm{Hf}$ & $3.8(15)$ & $1277 \rightarrow 93$ & $2,0_{2}^{+}$ & $86 \mathrm{Ha}$ \\
\hline${ }^{178} \mathrm{Hf}$ & $14(3)$ & $1496 \rightarrow 93$ & $2,0_{3}^{+}$ & $86 \mathrm{Ha}, 74 \mathrm{Ha}$ \\
\hline${ }^{182} \mathrm{~W}$ & $3.5(3)$ & $1257 \rightarrow 100$ & $2,0_{2}^{+}$ & \\
\hline${ }^{184} \mathrm{~W}$ & $2.6(5)$ & $1121 \rightarrow 111$ & $2,0_{2}^{+}$ & $74 \mathrm{Mc}$ \\
\hline${ }^{228} \mathrm{Th}$ & $2.4(3)$ & $1153 \rightarrow 969$ & $2,2_{2}^{+}$ & \\
\hline${ }^{230} \mathrm{Th}$ & $50(20)$ & $678 \rightarrow 53$ & $2,0_{2}^{+}$ & \\
\hline${ }^{232} \mathrm{Th}$ & $63\left(\begin{array}{c}+53 \\
-37\end{array}\right)$ & $774 \rightarrow 49$ & $2,0_{2}^{+}$ & $93 \mathrm{Mc}$ \\
\hline${ }^{238} \mathrm{U}$ & $176\left({ }_{-32}^{+36}\right)$ & $1037 \rightarrow 45$ & $2,0_{2}^{+}$ & $94 \mathrm{Mc}$ \\
\hline${ }^{238} \mathrm{Pu}$ & $180(110)$ & $983 \rightarrow 44$ & $2,0_{2}^{+}$ & \\
\hline${ }^{238} \mathrm{U}$ & $1.7 \times 10^{-6}$ & $2558 \rightarrow 0$ & $0,0_{\text {fission }}^{+}$ & \\
\hline
\end{tabular}

a. All $\rho^{2}$ (E0) values have been evaluated by the authors using data given in the relevant Nuclear Data Sheets together with the cited references.

$\begin{array}{ll}78 \mathrm{Gr} & \text { R.C.Greenwood et al., Nucl. Phys. A304, } 327 \\ 82 \mathrm{Ro} & \text { R.M.Ronningen et al. Phys. Rev. C26, } 97 \\ 68 \mathrm{Gr} & \text { T.Grotdal et al., Nucl. Phys. A110, } 385 \\ 97 \mathrm{Ga} & \text { P.E.Garrett et al., Phys. Lett. B400, } 250 \\ 98 \mathrm{Le} & \text { H.Lehmann et al., Phys. Rev. C57, 569 } \\ 81 \mathrm{Da} & \text { W.F.Davidson et al., J.Phys. G7, } 455 \\ 66 \mathrm{Gr} & \text { R.Graetzer et al., Nucl. Phys. 76, } 1 \\ 70 \mathrm{Ch} & \text { A.Charvet et al., Nucl. Phys. A156, } 276 \\ 72 \mathrm{Ca} & \text { D.C.Camp and F.M.Bernthal, Phys. Rev. C6, } 1040 \\ 88 \mathrm{Su} & \text { A.R.H.Subber et al., J.Phys. G14, } 87 \\ 85 \mathrm{Ge} & \text { W.Gelletly et al., J.Phys. G11, 1055 } \\ 75 \mathrm{Ca} & \text { M.H.Cardoso et al., Z.Phys. A272, } 13 \\ 71 \mathrm{Ch} & \text { A.Charvet et al., J.Phys. (Paris) } \mathbf{3 2 ,} 359 \\ 86 \mathrm{Ha} & \text { A.M.I.Hague et al., Nucl. Phys. A455, 231 } \\ 74 \mathrm{Ha} & \text { J.H.Hamilton et al., Phys. Rev. C10, 2540 } \\ 74 \mathrm{Mc} & \text { D.J.McMillan et al., Nucl. Phys. A223, 29 } \\ 93 \mathrm{Mc} & \text { F.K.Mc Gowan and W.T.Milner, Nucl. Phys. A562, } 241 \\ 94 \mathrm{Mc} & \text { F.K.Mc Gowan and W.T.Milner, Nucl. Phys. A571, } 569\end{array}$


Table 4. Excitation energies, $\rho^{2}(E 0)$ values in milliunits as well as reduced $\rho^{2}(E 0)$ values (multiplied by $A^{2 / 3}$ ) in selected ${ }^{a}$ light nuclei and compared to nuclei with the largest reduced $\rho^{2}(E 0)$ values (cf. Table 1$)$. The data for light nuclei are taken from ${ }^{114}$.

\begin{tabular}{cccc}
\hline \hline Nucleus & $E_{x}\left(0^{+}\right)(\mathrm{MeV})$ & $\rho^{2}(E 0) \times 10^{3}$ & $\rho^{2}(E 0) \times 10^{3} \cdot A^{2 / 3}$ \\
\hline${ }^{96} \mathrm{Sr}$ & 1.465 & $185(50)$ & 3880 \\
${ }^{20} \mathrm{Ne}$ & 7.191 & $430(160)$ & 3160 \\
${ }^{150} \mathrm{Sm}$ & 0.740 & $100(40)$ & 2820 \\
${ }^{12} \mathrm{C}$ & 7.655 & $530(40)$ & 2780 \\
${ }^{102} \mathrm{Mo}$ & 0.697 & $120(50)$ & 2620 \\
${ }^{18} \mathrm{O}$ & 3.652 & $370(80)$ & 2540 \\
${ }^{24} \mathrm{Mg}$ & 6.433 & $305(40)$ & 2440 \\
${ }^{28} \mathrm{Si}$ & 4.980 & $265(40)$ & 1630 \\
${ }^{42} \mathrm{Ca}$ & 1.837 & $135(12)$ & 1500 \\
${ }^{44} \mathrm{Ca}$ & 1.884 & $120(40)$ & 870 \\
${ }^{16} \mathrm{O}$ & 6.049 & $137(10)$ & 300 \\
${ }^{40} \mathrm{Ca}$ & 3.353 & $26(1)$ & 190 \\
${ }^{38} \mathrm{Ar}$ & 3.377 & $17(2)$ & \\
\hline
\end{tabular}

${ }^{a}$ These nuclei possess the largest $\rho^{2}(\mathrm{E} 0)$ values known, or are light nuclei that exhibit (see, e.g., ${ }^{5}$ ) shape coexistence. 